\title{
Soft-core meson-baryon interactions. I. One-hadron-exchange potentials
}

\author{
H. Polinder ${ }^{1,2}$ and Th. A. Rijken ${ }^{1}$ \\ ${ }^{1}$ Institute for Theoretical Physics, Radboud University Nijmegen, Nijmegen, The Netherlands \\ ${ }^{2}$ Forschungszentrum Jülich, Institut für Kernphysik (Theorie), D-52425 Jülich, Germany
}

(Received 30 May 2005; published 30 December 2005)

\begin{abstract}
The Nijmegen soft-core model for the pseudoscalar meson-baryon interaction is derived, analogous to the Nijmegen $N N$ and $Y N$ models. The interaction Hamiltonians are defined and the resulting amplitudes for onemeson exchange and one-baryon exchange in momentum space are given for the general mass case. The partial wave projection is carried through and explicit expressions for the momentum space partial wave meson-baryon potentials are presented.
\end{abstract}

DOI: 10.1103/PhysRevC.72.065210

PACS number(s): 12.39.Pn, 21.30.-x, 13.75.Gx, 13.75.Jz

\section{INTRODUCTION}

Strong interactions between mesons and baryons, in particular for pion-nucleon, kaon-nucleon, and antikaon-nucleon interactions, have been the subject of investigation for some decades, experimentally as well as theoretically.

Numerous scattering experiments have been performed to investigate the pion-nucleon interaction. The empirical phase shifts are obtained from a partial wave (PW) analysis of the scattering observables, which judges the consistency with general principles (unitarity, analyticity, crossing symmetry, etc.) of the scattering data, and which provides a compact representation of these data. Although at first sight in principle an infinite number of phase shifts need to be determined from the data, the strong interactions are short-ranged and only the lower partial wave phase shifts will suffice. In constructing theoretical models for these interactions, it is usually much more economical to use the results of a PW analysis than the scattering observables themselves. Different pion-nucleon PW analyses [1-3] give quite accurate and consistent results. The most recent pion-nucleon $\mathrm{PW}$ analysis has been performed by Arndt et al. [2], to which we refer for more information on the current pion-nucleon scattering database; see also Ref. [4].

However, the situation for the kaon-nucleon interaction is different from that for the pion-nucleon interaction. The kaonnucleon scattering observables are known to less accuracy, especially at low energies, owing to the relatively low flux of the kaon beams. Consequently, the different kaon-nucleon phase shift analyses give results that are not quite accurate and may not be totally consistent. The most recent kaon-nucleon PW analysis has been performed by Hyslop et al. [5], where much information on the kaon-nucleon scattering database can be found.

This lack of empirical knowledge makes it impossible to construct realistic theoretical kaon-nucleon models by using as input only information from $K N$ data.

Recently there has been an increase of interest in the kaon-nucleon and antikaon-nucleon interactions. An exotic resonance, the so-called penta-quark, in the isospin-zero kaon-nucleon system has been observed [6]; this experiment, however, was not a simple scattering experiment and a resonance has never been seen in the present kaon-nucleon scattering data.
The construction of new $K$ factories at the Japan Proton Accelerator Research Complex (J-PARC), and at GSI (FAIR) in Germany, will hopefully change the experimental situation drastically. One of the major beams of these new accelerators will be kaon beams, having a much higher intensity ( $\sim 10$ times) than that of presently available kaon beams at, for example, Brookhaven National Laboratory and KEK. Therefore, in the near future much more and accurate experimental data on the kaon-nucleon and antikaon-nucleon interaction can be expected. Other new scattering data could be delivered by the DA $\Phi$ NE facility at Frascati [7]. These activities will give much stronger constraints on kaon-nucleon models and a better understanding of the role of $\mathrm{SU}_{f}(3)$ in meson-baryon interactions. Akaishi and Yamazaki [8] have investigated the possibility of nuclear antikaon bound states in nuclei in the framework of the Brueckner-HartreeFock theory using a simple phenomenological antikaonnucleon model. Such a state has indeed been observed experimentally [9].

In view of these experimental and theoretical developments it is rather timely to construct theoretical kaon-nucleon models as realistically as possible, and this work is an attempt to do so.

The subject of this work is the construction of a dynamical model for the pion-nucleon $(\pi N)$ and kaon-nucleon $\left(K^{+} N\right)$ interactions. In two papers we describe the so-called Nijmegen soft-core meson-baryon model (NSC model) and report on the results obtained so far. First a soft-core meson- and baryonexchange model for the $\pi N$ interaction is derived, showing that the soft-core approach of the Nijmegen group is not only successful for baryon-baryon $(N N$ and $Y N)$ interactions but also for meson-baryon interactions. The rich and accurate $\pi N$ scattering database is used to determine the nonstrange coupling constants. Several other $\pi N$ models already exist and the NSC $\pi N$ model, besides having value in its own right, mainly serves as a natural starting point for the construction of the NSC $K^{+} N$ model. This $K^{+} N$ model is an $\mathrm{SU}_{f}(3)$ extension of the NSC $\pi N$ model, similar to the successful Nijmegen soft-core one-boson-exchange nucleon-nucleon and hyperon-nucleon models [10]. In this way many parameters in the NSC $K^{+} N$ model are determined by the NSC $\pi N$ model, and the lack of accurate $K^{+} N$ data can be overcome partially. 


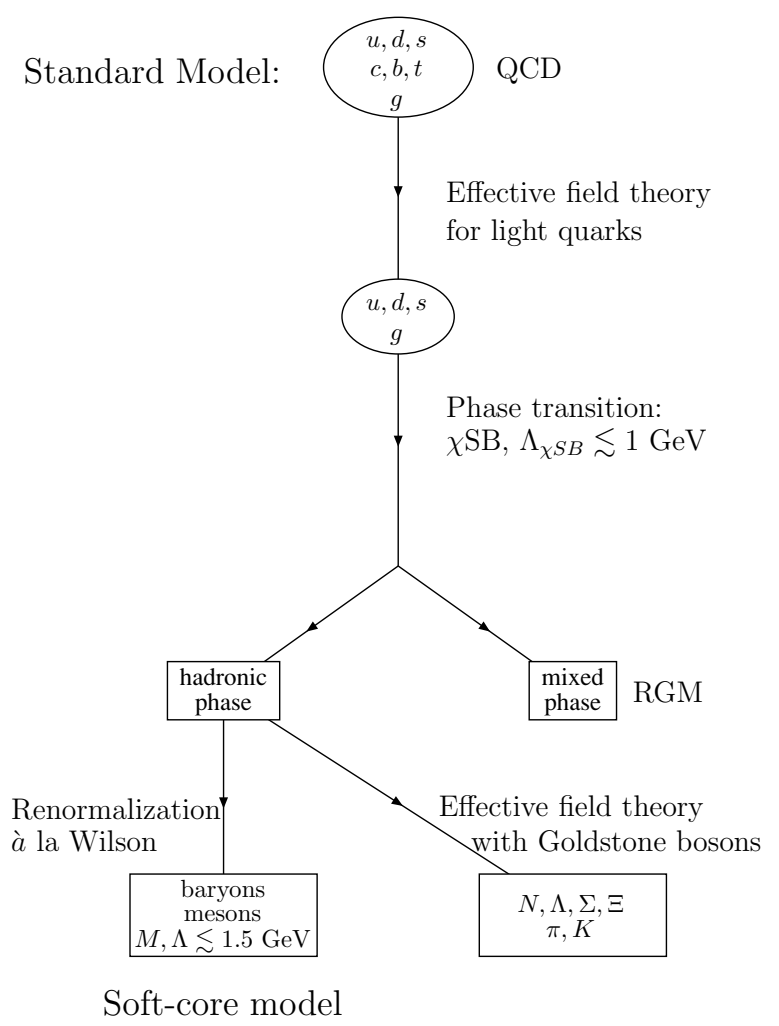

FIG. 1. Overview of the theoretical basis for soft-core mesonbaryon interactions.

In concept, the approach for the strong low- and intermediate-energy hadron-hadron interactions [11], used by the Nijmegen group, is schematically outlined in Fig. 1. The starting point is the Standard Model, in which strong interactions occur between the six quarks and the gluons. Next, one can integrated out the heavier quarks, to arrive at an effective QCD for the light quarks $(u, d, s)$ only. As is generally accepted, the vacuum of QCD becomes unstable for momenta transfer $q^{2} \leqslant \Lambda_{\chi \mathrm{SB}}^{2} \simeq 1 \mathrm{GeV}^{2}$ and the chiral symmetry is broken spontaneously $(\chi \mathrm{SB})$. The vacuum goes through a phase transition and generates constituent quark masses $\left(m_{q} \approx 300 \mathrm{MeV}\right)$ and reduces the strong coupling constant $\alpha_{s}$. The pseudoscalar mesons are viewed as the Nambu-Goldstone bosons originating from the $\chi \mathrm{SB}$, which makes it natural to assume the presence of a meson cloud around the constituent quarks.

This framework provides a natural basis for an approach to the interaction between mesons and baryons using effective baryon-meson Lagrangians. At low and intermediate energies we do not consider a mixed phase of hadrons and quarks, as is done by others using the resonating-group method (RGM) [12], but restrict ourselves to the hadronic phase only. Furthermore, heavy baryons and mesons can be viewed as being integrated out, using for example the renormalization method in the manner of Wilson [13], and an effective field theory, with meson and baryon masses with $M \leqslant 1.5 \mathrm{GeV}$, results. In this work, this general picture is invoked in the construction of a soft-core meson-baryon model for low- and intermediate-energy interactions.
In the NSC model the one-meson-exchange and onebaryon-exchange potentials are obtained from field theoretical Feynman diagrams in momentum space using effective interaction Hamiltonians; together with the meson-baryon Green's function they constitute the kernel of the two-particle integral equation for the amplitude, which is a three-dimensional reduction of the fully covariant (four-dimensional) BetheSalpeter equation [14]. Alternatively, one could view this work in the framework of the covariant perturbation theory as formulated by Kadyshevsky [15]. Here the particles, also in the intermediate states, remain on the mass shell, and pair suppression can be implemented in a covariant way. Moreover, the three-dimensional integral equation obtained in the Kadyshevsky scheme has exactly the same form as used in this work.

Form factors of the Gaussian type are introduced to take into account the extended size of the hadrons and to make the integral equation of the Fredholm type. The Coulomb interaction, which plays a role at very low energies only and is important in charge-symmetry-breaking (CSB) studies, will be neglected in this work. The integral equation for the amplitude is solved on the partial wave basis; in this way only one-dimensional integrals need to be performed to find the amplitude and the corresponding scattering observables or phase shifts for each partial wave.

We present this work in two papers. In general, this first paper, referred to as I, contains a description of the theory; the second paper, referred to as paper II [16], gives the results for $\pi N$ and $K^{+} N$.

The contents of this first paper are as follows. The definition of the field theoretical one-meson-exchange and one-baryonexchange potentials in the context of a three-dimensional integral equation, a relativistic generalization of the LippmannSchwinger equation, is reviewed in Sec. II. We introduce the usual potential forms in Pauli-spinor space, where we include the central (C) and the spin-orbit ( $\mathrm{SO}$ ) potentials, which are the only relevant potentials in case of spin-0 spin-1/2 interactions. The relations between the relativistic and center-of-mass amplitudes are also given.

The integral equation for the amplitude is solved on the partial wave basis to find the partial wave phase shifts, which are compared with the empirical phase shifts. Therefore we perform the basic partial wave projections, in particular those for the spinor invariants, in Sec. III. And the relations among the partial wave amplitudes, the phase shifts, and the scattering observables- $\sigma, d \sigma / d \Omega$, and $P$ - for spin- 0 spin-1/2 scattering are given. The partial wave basis is chosen according to the convention of [17].

The effective baryon-baryon-meson and meson-mesonmeson interaction Hamiltonians, from which the one-mesonexchange and one-baryon-exchange Feynman diagrams are derived, are given in Sec. IV. The explicit expressions for the momentum-space Feynman diagrams for scalar-meson, vector-meson, tensor-meson, and baryon exchanges for general baryon and meson masses as well as their partial wave projections are also listed in this section.

In the appendices, details are given on the calculation of the partial wave matrix elements (Appendix A), the one-particleexchange Feynman diagrams (Appendix B), and the expansion 
coefficients $X, Y$, and $Z$ of the partial wave potentials in $x=$ $\cos \theta$ for the different exchanges (Appendix C).

For results and a discussion, we refer to paper II.

\section{THE MESON-BARYON POTENTIAL AND AMPLITUDE}

The field theoretical one-particle-exchange meson-baryon potentials in the context of a two-particle equation are defined in this section for the case of $J^{\mathrm{PC}}=0^{-+}$mesons and $J^{P}=1 / 2$ baryons. We approximate the Bethe-Salpeter equation by assuming "dynamical pair suppression"; hence by neglecting the propagation of negative-energy states and integrating out the time variable, we end up with a three-dimensional integral equation for the meson-baryon amplitude in the center-of-mass (c.m.) system. The relations between the center of mass and the relativistic amplitudes are given in the last part of this section.

\section{A. Kinematics and relativistic amplitudes}

We consider the meson-baryon or more specific the $\pi N$ and $K^{+} N$ reactions

$$
\mathcal{P}_{i}(q)+\mathcal{B}_{i}(p, s) \rightarrow \mathcal{P}_{f}\left(q^{\prime}\right)+\mathcal{B}_{f}\left(p^{\prime}, s^{\prime}\right),
$$

where $\mathcal{P}$ stands for the pseudoscalar mesons, and $\mathcal{B}$ stands for the baryons. We will refer to $\mathcal{P}_{i}$ and $\mathcal{P}_{f}$ as particles 1 and 3 and to $\mathcal{B}_{i}$ and $\mathcal{B}_{f}$ as particles 2 and 4. The four-momentum of particle $i$ is $p_{i}=\left(E_{i}, \mathbf{p}_{i}\right)$, where $E_{i}=\sqrt{\mathbf{p}_{i}^{2}+M_{i}^{2}}$ is the energy and $M_{i}$ is the mass of particle $i$. In our convention the transition amplitude matrix $M$ is related to the $S$ matrix via

$$
\langle f|S| i\rangle=\langle f \mid i\rangle-i(2 \pi)^{4} \delta^{4}\left(P_{f}-P_{i}\right)\langle f|M| i\rangle,
$$

in this convention a negative potential corresponds to attraction and a positive potential to repulsion. Here $P_{i}=p+q$ and $P_{f}=p^{\prime}+q^{\prime}$ represent the total four-momenta for the initial state $|i\rangle$ and the final state $|f\rangle$. The latter refer to the twoparticle states, which we normalize in the following way (see, e.g., Refs. [18,19]):

$$
\begin{aligned}
\left\langle\mathbf{p}_{1}^{\prime}, \mathbf{p}^{\prime}{ }_{2} \mid \mathbf{p}_{1}, \mathbf{p}_{2}\right\rangle= & (2 \pi)^{3} 2 E\left(\mathbf{p}_{1}\right) \delta^{3}\left(\mathbf{p}_{1}^{\prime}-\mathbf{p}_{1}\right) \\
& \times(2 \pi)^{3} 2 E\left(\mathbf{p}_{2}\right) \delta^{3}\left(\mathbf{p}_{2}^{\prime}-\mathbf{p}_{2}\right) .
\end{aligned}
$$

With this normalization, the unpolarized differential cross section in the c.m. system is given by

$$
\left(\frac{d \sigma}{d \Omega}\right)_{\mathrm{c} . \mathrm{m} .}=\frac{p_{f}}{p_{i}} \frac{1}{2} \sum\left|\frac{\langle f|M| i\rangle}{8 \pi \sqrt{s}}\right|^{2},
$$

where $\sum$ stands for the summation over the spin of the final baryon.

In this work, the scattering particles are always on the mass shell (i.e., $p_{i}^{2}=m_{i}^{2}$ ), so parity conservation and Lorentz invariance implies that the matrix elements of the $M$ operator for meson-baryon interactions, which is a $4 \times 4$ matrix sandwiched between Dirac spinors, can be written in terms of two independent amplitudes

$$
\begin{aligned}
\langle f|M| i\rangle= & \bar{u}_{B^{\prime}}\left(\mathbf{p}^{\prime}, s_{f}\right)\left[A_{f i}(s, t, u)\right. \\
& \left.+\frac{q^{\prime}+\not q}{2} B_{f i}(s, t, u)\right] u_{B}\left(\mathbf{p}, s_{i}\right),
\end{aligned}
$$

where $f$ and $i$ stand for the two-particle channels $\pi N, K^{+} N$, etc.... In the Dirac spinors $s_{f}, s_{i}$ are the magnetic spin variables, which will be specified later. The functions $A_{f i}(s, t, u)$ and $B_{f i}(s, t, u)$ are Lorentz scalars and depend on the Mandelstam invariants

$$
\begin{aligned}
s & =(p+q)^{2}=\left(p^{\prime}+q^{\prime}\right)^{2}, \\
t & =\left(q^{\prime}-q\right)^{2}=\left(p-p^{\prime}\right)^{2}, \\
u & =\left(p-q^{\prime}\right)^{2}=\left(p^{\prime}-q\right)^{2},
\end{aligned}
$$

which satisfy the well-known (on-mass-shell) relation $s+t+$ $u=\sum_{i=1}^{4} m_{i}^{2}$. The total and relative four-momenta $\left(P_{c}\right.$ and $k_{c}$ ) of the initial, final, and intermediate channel $(c=i, f, n)$ are defined by

$$
P_{c}=p_{c}+q_{c}, \quad k_{c}=\mu_{c, 2} p_{c}-\mu_{c, 1} q_{c},
$$

where the weights are arbitrary apart from the condition $\mu_{c, 1}+$ $\mu_{c, 2}=1$. For each channel the four-momenta of the baryons and pseudoscalar mesons $\left(p_{c}\right.$ and $\left.q_{c}\right)$ in terms of $P_{c}$ and $k_{c}$ are

$$
p_{c}=\mu_{c, 1} P_{c}+k_{c}, \quad q_{c}=\mu_{c, 2} P_{c}-k_{c} .
$$

In this work we will use $\mu_{c, 1}=\mu_{c, 2}=1 / 2$. In the c.m. system we have for on-mass-shell momenta

$$
\begin{array}{ll}
p_{c}=\left(E\left(\mathbf{p}_{c}\right), \mathbf{p}_{c}\right), & q_{c}=\left(\mathcal{E}\left(\mathbf{p}_{c}\right),-\mathbf{p}_{c}\right), \\
P_{c}=\left(W_{c}, \mathbf{0}\right), & k_{c}=\left(\mu_{2} E\left(\mathbf{p}_{c}\right)-\mu_{1} \mathcal{E}\left(\mathbf{p}_{c}\right), \mathbf{p}_{c}\right),
\end{array}
$$

where the total energy is $W_{c}=\sqrt{s}=E\left(\mathbf{p}_{c}\right)+\mathcal{E}\left(\mathbf{p}_{c}\right)$. Obviously the relative three-momentum is equal to the c.m. three-momentum of the baryon.

In general Feynman diagrams, in particular in the Green's functions, the particles are off the mass shell. In the following the three-momenta for the initial and the final states are denoted, respectively, by $\mathbf{q}_{i}$ and $\mathbf{q}_{f}$. Because of translation invariance $P_{i}=P_{f}$ and so $\sqrt{s}=W_{i}=W_{f}$. As introduced here, the total energies in the c.m. system are $W_{i}=E\left(\mathbf{q}_{i}\right)+$ $\mathcal{E}\left(\mathbf{q}_{i}\right)$ and $W_{f}=E\left(\mathbf{q}_{f}\right)+\mathcal{E}\left(\mathbf{q}_{f}\right)$.

\section{B. Relativistic two-particle equations}

The Bethe-Salpeter equation, a full two-particle relativistic scattering equation, for the $\mathcal{M}$ amplitudes reads

$$
\begin{aligned}
\mathcal{M}_{f i}\left(q_{f}, q_{i} ; P\right)= & \mathcal{M}_{f i}^{\mathrm{irr}}\left(q_{f}, q_{i} ; P\right)+\sum_{n} \int d^{4} k_{n} \\
& \times \mathcal{M}_{f n}^{\mathrm{irr}}\left(q_{f}, k_{n} ; P\right) G\left(k_{n}, P\right) \mathcal{M}_{n i}\left(k_{n}, q_{i} ; P\right),
\end{aligned}
$$

where the interaction kernel is denoted by $\mathcal{M}^{\text {irr }}, G$ is the two-particle Green's function, and the summation $\sum_{n}$ is over all intermediate two-particle channels coupled to the initial and final states. The contributions to the kernel $\mathcal{M}^{\text {irr }}$ come from the meson-baryon irreducible Feynman diagrams. The reducible diagrams are generated by the integral equation. In deriving Eq. (2.10) the integration over the momenta of the intermediate particles can be replaced by an integration over the total and relative momenta $\iint d^{4} p_{n} d^{4} q_{n} \rightarrow \iint d^{4} P_{n} d^{4} k_{n}$. Then, using the conservation of the total four-momentum, one can 
perform $\int d^{4} P_{c}$ and separate an overall $(2 \pi)^{4} \delta^{4}\left(P_{f}-P_{i}\right)$ factor. The meson-baryon Green's function is given in terms of the one-particle Green's functions

$$
\begin{aligned}
G\left(k_{n}, P\right)= & \frac{i}{(2 \pi)^{4}}\left[\frac{1}{\gamma\left(\mu_{n, 1} P+k\right)-M_{n}}\right] \\
& \times\left[\frac{1}{\left(\mu_{n, 2} P-k\right)^{2}-m_{n}^{2}}\right] .
\end{aligned}
$$

It is instructive to separate the positive- and the negative-energy components of the propagator. For that purpose, we rewrite the one-particle propagators as follows. For the spin-1/2 baryons the off-mass-shell propagator can be written in terms of the Dirac spinors as

$\frac{p p+M}{p^{2}-M^{2}+i \delta}=\frac{M}{E(\mathbf{p})}\left[\frac{\Lambda_{+}(\mathbf{p})}{p^{0}-E(\mathbf{p})+i \delta}-\frac{\Lambda_{-}(-\mathbf{p})}{p^{0}+E(\mathbf{p})-i \delta}\right]$,

where the projection operators $\Lambda_{+}(\mathbf{p})$ and $\Lambda_{-}(\mathbf{p})$ on the positive- and negative-energy states are [20]

$$
\begin{aligned}
& \Lambda_{+}(\mathbf{p})=\sum_{s} \frac{u(\mathbf{p}, s) \otimes \bar{u}(\mathbf{p}, s)}{2 M}=\frac{\not p+M}{2 M}, \\
& \Lambda_{-}(\mathbf{p})=-\sum_{s} \frac{v(\mathbf{p}, s) \otimes \bar{v}(\mathbf{p}, s)}{2 M}=\frac{-\not p+M}{2 M},
\end{aligned}
$$

and $u(\mathbf{p}, s)$ and $v(\mathbf{p}, s)$ are the Dirac spinors for spin-1/2 particles, which are on the mass shell by definition. For the meson propagator similar to Eq. (2.12) one has the identity

$$
\begin{aligned}
\frac{1}{q^{2}-m^{2}+i \delta}= & \frac{1}{2 \mathcal{E}(\mathbf{q})}\left[\frac{1}{q^{0}-\mathcal{E}(\mathbf{q})+i \delta}\right. \\
& \left.-\frac{1}{q^{0}+\mathcal{E}(\mathbf{q})-i \delta}\right] .
\end{aligned}
$$

Then, in the c.m. system, where $\mathbf{P}=0$ and $P_{0}=W$, the meson-baryon Green's function can be written as

$$
\begin{aligned}
G\left(k_{n}, P\right)= & \frac{i}{(2 \pi)^{4}}\left[\frac{M}{2 E\left(\mathbf{k}_{n}\right) \mathcal{E}\left(\mathbf{k}_{n}\right)}\right] \\
& \times\left[\frac{\Lambda_{+}\left(\mathbf{k}_{n}\right)}{\mu_{n, 1} W+k_{n}^{0}-E\left(\mathbf{k}_{n}\right)+i \delta}\right. \\
& \left.-\frac{\Lambda_{-}\left(-\mathbf{k}_{n}\right)}{\mu_{n, 1} W+k_{n}^{0}+E\left(\mathbf{k}_{n}\right)-i \delta}\right] \\
& \times\left[\frac{1}{\mu_{n, 2} W-k_{n}^{0}-\mathcal{E}\left(\mathbf{k}_{n}\right)+i \delta}\right. \\
& \left.-\frac{1}{\mu_{n, 2} W-k_{n}^{0}+\mathcal{E}\left(\mathbf{k}_{n}\right)-i \delta}\right] .
\end{aligned}
$$

By multiplying out Eq. (2.15) and writing the ensuing terms using an obvious shorthand notation, the contribution of the different propagating components is displayed fully:

$$
G\left(k_{n}, P\right)=G^{+}\left(k_{n}, W\right)+G^{-}\left(k_{n}, W\right),
$$

where the superscripts $(+)$ and $(-)$ indicate the positiveand negative-energy baryon states. Considering similarly the amplitudes $M_{i j}^{\beta, \alpha}$, one gets

$$
\begin{aligned}
& M_{i j}^{+,+}=\bar{u}_{B^{\prime}}\left(\mathbf{p}_{f}, s_{f}\right) \mathcal{M}_{i j} u_{B}\left(\mathbf{p}_{i}, s_{i}\right), \\
& M_{i j}^{+,-}=\bar{u}_{B^{\prime}}\left(\mathbf{p}_{f}, s_{f}\right) \mathcal{M}_{i j} v_{B}\left(\mathbf{p}_{i}, s_{i}\right), \ldots,
\end{aligned}
$$

where the subscripts $i$ and $j$ refer to the different two-particle channels. One obtains from Eqs. (2.10), (2.16), and (2.17) the full relativistic scattering equation

$$
\begin{aligned}
M_{f i}^{\beta, \alpha}\left(q_{f}, q_{i} ; P\right)= & \left(M^{\mathrm{irr}}\right)_{f i}^{\beta, \alpha}\left(q_{f}, q_{i} ; P\right) \\
& +\sum_{n} \int d^{4} k_{n}\left(M^{\mathrm{irr}}\right)_{f n}^{\beta, \gamma}\left(q_{f}, k_{n} ; P\right) \\
& \times G_{n}^{\gamma}\left(k_{n}, P\right) M_{n i}^{\gamma, \alpha}\left(k_{n}, q_{i} ; P\right) .
\end{aligned}
$$

In all we have $2^{2}=4$ amplitudes, which are coupled as illustrated in Eq. (2.18).

The complexity of the previous equation can be reduced considerably if we assume dynamical pair suppression, that is, if we neglect the contribution of negative-energy states. Then the full scattering equation, Eq. (2.18), for $\alpha=+$ and $\beta=+$, reduces to the four-dimensional integral equation

$$
\begin{aligned}
M_{f i}^{+,+}\left(q_{f}, q_{i} ; P\right)= & \left(M^{\mathrm{irr}}\right)_{f i}^{+,+}\left(q_{f}, q_{i} ; P\right) \\
& +\sum_{n} \int d^{4} k_{n}\left(M^{\mathrm{irr}}\right)_{f n}^{+,+}\left(q_{f}, k_{n} ; P\right) \\
& \times G_{n}^{+}\left(k_{n}, P\right) M_{n i}^{+,+}\left(k_{n}, q_{i} ; P\right),
\end{aligned}
$$

with the positive-energy Green's function

$$
\begin{aligned}
G_{n}^{+}\left(k_{n}, P\right) \approx & \frac{i}{(2 \pi)^{4}}\left[\frac{1}{4 E\left(\mathbf{k}_{n}\right) \mathcal{E}\left(\mathbf{k}_{n}\right)}\right] \\
& \times \frac{1}{\left[\mu_{1} W+k_{n}^{0}-E\left(\mathbf{k}_{n}\right)+i \delta\right]} \\
& \times \frac{1}{\left[\mu_{2} W-k_{n}^{0}-\mathcal{E}\left(\mathbf{k}_{n}\right)+i \delta\right]} .
\end{aligned}
$$

We note that this simplification in principle brings about a hopefully tolerable breach of relativistic invariance. However, in Feynman diagrams particles go off the mass shell, and the off-mass-shell behavior is not really known for mesons and baryons, certainly not if a truncated kernel is used, which is always the case. Then it might be better to allow positiveenergy states only.

\section{Three-dimensional two-particle equations}

Three-dimensional integral equations for the amplitudes can be derived in various ways. The methods assume twoparticle unitarity as a basic ingredient. The derivation for the meson-baryon systems follows the same procedure as that for the baryon-baryon channels. For the latter see, for example, Ref. [21-23]. In [24] the derivation is based entirely on two-particle unitarity and the analyticity properties of the amplitudes, using the $N / D$ formalism. In the latter approach, in essence, the Regge pole nature of meson exchange can be apprehended most easily. 


\section{On-mass-shell approximation}

The simplest way to reduce the four-dimensional integral equation, Eq. (2.19), to a three-dimensional one is to put the intermediate particles on the mass shell [i.e., $p_{n}^{0}=$ $\left.E\left(\mathbf{k}_{n}\right)=\sqrt{\mathbf{k}_{n}^{2}+M_{n}^{2}} \cdot q_{n}^{0}=\mathcal{E}\left(\mathbf{k}_{n}\right)=\sqrt{\mathbf{k}_{n}^{2}+m_{n}^{2}}\right]$. It can readily be shown from Eq. (2.8) that the zero components of the relative and total momenta $k_{n}$ and $P_{n}$ are given by

$$
\begin{aligned}
k_{n}^{0} & =\mu_{n, 2} E\left(\mathbf{k}_{n}\right)-\mu_{n, 1} \mathcal{E}\left(\mathbf{k}_{n}\right), \\
P_{n}^{0} & =E\left(\mathbf{k}_{n}\right)+\mathcal{E}\left(\mathbf{k}_{n}\right) .
\end{aligned}
$$

If we neglect the $k_{n}^{0}$ dependence of the amplitudes, and evaluate them at the value given by Eq. (2.21), the dependence of the four-dimensional equation on $k_{n}^{0}$ only occurs in the Green's function, and the $k_{n}^{0}$ integration of the Green's function can be done. We can define the $k_{n}^{0}$-independent amplitudes

$$
\begin{aligned}
T_{n i}\left(\mathbf{k}_{n}, \mathbf{q}_{i} ; W\right) & =M_{n i}^{+,+}\left(\tilde{k}_{n}, q_{i} ; P\right), \\
V_{f n}\left(\mathbf{q}_{f}, \mathbf{k}_{n} ; W\right) & =\left(M^{\mathrm{irr}}\right)_{f n}^{+,+}\left(q_{f}, \tilde{k}_{n} ; P\right),
\end{aligned}
$$

where $\tilde{k}_{n}^{0}=\mu_{n, 2} E\left(\mathbf{k}_{n}\right)-\mu_{n, 1} \mathcal{E}\left(\mathbf{k}_{n}\right)$. Now, the $k_{n}^{0}$ integration in Eq. (2.19) can be carried through, which leads to

$$
\begin{aligned}
G_{0}\left(\mathbf{k}_{n} ; W\right)= & \int_{-\infty}^{\infty} d k_{n 0} G_{n}^{+}\left(k_{n} ; P\right) \\
= & \frac{1}{(2 \pi)^{3}} \frac{1}{4 E\left(\mathbf{k}_{n}\right) \mathcal{E}\left(\mathbf{k}_{n}\right)} \\
& \times \frac{1}{W-E\left(\mathbf{k}_{n}\right)-\mathcal{E}\left(\mathbf{k}_{n}\right)+i \delta} .
\end{aligned}
$$

The four-dimensional integral equation, Eq. (2.19), now results in the three-dimensional integral equation, which is also derived in [23],

$$
\begin{aligned}
T_{f i}\left(\mathbf{q}_{f}, \mathbf{q}_{i} ; W\right)= & V_{f i}\left(\mathbf{q}_{f}, \mathbf{q}_{i} ; W\right)+\sum_{n} \int \frac{d^{3} k_{n}}{(2 \pi)^{3}} \\
& \times V_{f n}\left(\mathbf{q}_{f}, \mathbf{k}_{n} ; W\right) G_{0}\left(\mathbf{k}_{n}, W\right) T_{n i}\left(\mathbf{k}_{n}, \mathbf{q}_{i} ; W\right) .
\end{aligned}
$$

The integral equation for the $T$ matrix, Eq. (2.24), is schematically given in Fig. 2.

We remark that the three-dimensional integral equation for the amplitude, Eq. (2.24), is here obtained as an approximation of the Bethe-Salpeter equation, but in the formulation of quantum field theory (QFT) as developed by Kadyshevsky [15] this integral equation is obtained without making any approximation. In this formulation of QFT all particles, in particular the intermediate particles, are always on the mass shell, in contrast to the formalism of Feynman. Hence a covariant form of pair suppression can be introduced phenomenologically.

Until this subsection the intermediate particles were in principle off the mass shell and the total four-momentum was

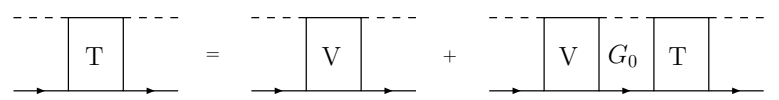

FIG. 2. Diagrammatic representation of the meson-baryon scattering equation (2.24). The solid line denotes the baryon and the dashed line denotes the meson. conserved. Now we have put the intermediate particles on the mass shell, but now in principle they are off the energy shell, which means that $W \neq E\left(\mathbf{k}_{n}\right)+\mathcal{E}\left(\mathbf{k}_{n}\right)$. And the total fourmomentum is not conserved, but the total three-momentum is conserved.

Note that if the intermediate state is on the energy shell [i.e., $W=E\left(\mathbf{k}_{n}\right)+\mathcal{E}\left(\mathbf{k}_{n}\right)$ ], the two poles of the Green's function, Eq. (2.20), coincide. The value of $k_{n}^{0}$ at which this "pinching" occurs is given by the on-mass-shell value, Eq. (2.21),

$$
k_{n}^{0}=\mu_{n, 2} E\left(\mathbf{k}_{n}\right)-\mu_{n, 1} \mathcal{E}\left(\mathbf{k}_{n}\right) \pm i \delta .
$$

The contribution to the integral around this value of $k_{n}^{0}$ will be dominant, owing to the pinching of the poles. This is the rationale for the "on-mass-shell approximation."

\section{Potentials for the three-dimensional integral equation}

To calculate cross sections or phase shifts we need to solve Eq. (2.24), which is a complex integral equation for the $T$ matrix, even for physical momenta. It is possible to transform Eq. (2.24) into a Lippmann-Schwinger equation (which can be Fourier-transformed into coordinate space). However, we do our calculations always in momentum space, so we do not need to solve the Lippmann-Schwinger equation but we will always solve Eq. (2.24).

Using rotational invariance and parity conservation we expand the $T$ matrix, which is a $2 \times 2$-matrix in Paulispinor space, into a complete set of Pauli-spinor invariants. Introducing the momentum vectors

$$
\mathbf{q}=\frac{1}{2}\left(\mathbf{q}_{f}+\mathbf{q}_{i}\right), \quad \mathbf{k}=\mathbf{q}_{f}-\mathbf{q}_{i}, \quad \hat{\mathbf{n}}=\hat{\mathbf{q}}_{i} \times \hat{\mathbf{q}}_{f},
$$

where $\mathbf{q}_{f}$ and $\mathbf{q}_{i}$ are the final and initial c.m. three-momenta, respectively, there are only two independent spinor invariants, $P_{\alpha}$, rotational invariant and invariant under parity transformations. We choose for the operators $P_{\alpha}$ in spin space

$$
P_{1}=1, \quad P_{2}=\sigma \cdot \mathbf{q}_{i} \times \mathbf{q}_{f},
$$

corresponding to the central and spin-orbit piece of the amplitude. Now the expansion of the $T$ matrix in spinor invariants reads

$$
\begin{aligned}
T & =\sum_{1}^{2} T_{\alpha}\left(\mathbf{q}_{f}^{2}, \mathbf{q}_{i}^{2}, \mathbf{q}_{f} \cdot \mathbf{q}_{i}\right) P_{\alpha} \\
& =f\left(\mathbf{q}_{f}, \mathbf{q}_{i}\right)+i g\left(\mathbf{q}_{f}, \mathbf{q}_{i}\right)(\boldsymbol{\sigma} \cdot \hat{\mathbf{n}}) .
\end{aligned}
$$

For the partial wave projection we found it convenient to rewrite the $T$ matrix in terms of the amplitudes $F$ and $G$ :

$$
T=F\left(\mathbf{q}_{f}, \mathbf{q}_{i}\right)+\left(\boldsymbol{\sigma} \cdot \hat{\mathbf{q}}_{f}\right) G\left(\mathbf{q}_{f}, \mathbf{q}_{i}\right)\left(\boldsymbol{\sigma} \cdot \hat{\mathbf{q}}_{i}\right) .
$$

The relation between the "spin-nonflip" and "spin-flip" amplitudes $f, g$ and the amplitudes $F, G$ is readily found to be

$$
F=f+\left(\hat{\mathbf{q}}_{f} \cdot \hat{\mathbf{q}}_{i}\right) g, \quad G=-g .
$$

The connection between the nonrelativistic amplitudes $F$ and $G$ defined in Eq. (2.29) and the relativistic amplitudes $A$ and $B$ defined in Eq. (2.5) can be obtained in a straightforward way using the explicit representation of the Dirac spinors, as will 
be shown in Sec. II D. Similar to Eq. (2.28) we expand the potentials $V$, so

$$
\begin{aligned}
V & =\sum_{1}^{2} V_{\alpha}\left(\mathbf{q}_{f}^{2}, \mathbf{q}_{i}^{2}, \mathbf{q}_{f} \cdot \mathbf{q}_{i}\right) P_{\alpha} \\
& =V_{C}\left(\mathbf{q}_{f}, \mathbf{q}_{i}\right)+i V_{S O}\left(\mathbf{q}_{f}, \mathbf{q}_{i}\right)(\boldsymbol{\sigma} \cdot \mathbf{n}) .
\end{aligned}
$$

\section{Lippmann-Schwinger equation}

To arrive at a Lippmann-Schwinger equation, one chooses a new Green's function $g(\mathbf{k}, W)$ that satisfies a dispersion relation in $\mathbf{p}^{2}(s)$ rather than in $s$ [21]. Then one obtains

$$
g\left(\mathbf{k}_{n}, W\right)=\frac{-1}{2\left[E\left(\mathbf{k}_{n}\right)+\mathcal{E}\left(\mathbf{k}_{n}\right)\right]}\left(\mathbf{k}_{n}^{2}-\mathbf{q}_{n}^{2}-i \delta\right)^{-1},
$$

where $\mathbf{q}_{n}$ is the on-energy-shell momentum. This Green's function is then used in the integral equation (2.24) instead of the Green's function $G_{0}\left(\mathbf{k}_{n}, W\right)$. So the corrections to $\langle f|W| i\rangle$ owing to the transformation of the Green's functions are neglected here; they are of higher order in the couplings and are usually discarded in a one-boson-exchange approach. With the substitution of $g$ for $G_{0}$, Eq. (2.23) becomes identical to Eq. (2.19) of [21]. From now on we follow Sec. II of [21] in detail. The transformation to the nonrelativistic normalization of the two-particle states leads to states with

$$
\begin{aligned}
\left(\mathbf{p}_{1}^{\prime}, s_{1}^{\prime} ; \mathbf{p}_{2}^{\prime}, s_{2}^{\prime} \mid \mathbf{p}_{1}, s_{1} ; \mathbf{p}_{2}, s_{2}\right)= & (2 \pi)^{6} \delta^{3}\left(\mathbf{p}_{1}^{\prime}-\mathbf{p}_{1}\right) \delta_{s_{1}^{\prime}, s_{1}} \\
& \times \delta^{3}\left(\mathbf{p}_{2}^{\prime}-\mathbf{p}_{2}\right) \delta_{s_{2}^{\prime}, s_{2}} .
\end{aligned}
$$

For these states we define the nonrelativistic $\mathcal{T}$ matrix

$$
(f|\mathcal{T}| i)=\frac{1}{\sqrt{4 \mu_{34}\left(E_{3}+E_{4}\right)}}\langle f|T| i\rangle \frac{1}{\sqrt{4 \mu_{12}\left(E_{1}+E_{2}\right)}},
$$

where $\mu_{12}$ and $\mu_{34}$ are the reduced masses for, respectively, the initial and final states. Then we get from Eq. (2.24) the Lippmann-Schwinger equation

$$
\begin{aligned}
(3,4|\mathcal{T}| 1,2)= & (3,4|\mathcal{V}| 1,2)+\sum_{n} \int \frac{d^{3} k_{n}}{(2 \pi)^{3}}\left(3,4|\mathcal{V}| n_{1}, n_{2}\right) \\
& \times \frac{2 \mu_{n_{1}, n_{2}}}{\mathbf{q}_{n}^{2}-\mathbf{k}_{n}^{2}+i \delta}\left(n_{1}, n_{2}|\mathcal{T}| 1,2\right)
\end{aligned}
$$

where the potential $\mathcal{V}$ is defined analogously to the $\mathcal{T}$ matrix, Eq. (2.34). If, in the low-energy approximation, the energies are expanded in terms of the momenta squared, the LippmannSchwinger equation in momentum space can in principle be Fourier-transformed into the equivalent Schrödinger equation in configuration space. However, our calculations are always in momentum space, so we always solve Eq. (2.24).

\section{Relation between relativistic and c.m. amplitudes}

The relation between the relativistic amplitudes $A$ and $B$ and the nonrelativistic amplitudes $F$ and $G$ is found by making use of the representation of the Dirac spinors [20]. Since in the three-dimensional integral equation, Eq. (2.24), off-energyshell amplitudes appear, we now distinguish between the c.m. energies of the final and initial states, defined by

$$
W_{f}^{2} \equiv s_{f}=\left(p^{\prime}+q^{\prime}\right)^{2}, \quad W_{i}^{2} \equiv s_{i}=(p+q)^{2} .
$$

Then, a straightforward calculation of the operators 1 and $\emptyset$ between Dirac spinors gives the corresponding operators between Pauli spinors:

$$
\begin{aligned}
\bar{u}\left(\mathbf{p}_{f}, s_{f}\right) u\left(\mathbf{p}_{i}, s_{i}\right)= & \sqrt{\left(E_{f}+M_{f}\right)\left(E_{i}+M_{i}\right)} \chi_{f}^{\dagger} \\
& \times\left[1-\frac{\boldsymbol{\sigma} \cdot \mathbf{p}_{f} \boldsymbol{\sigma} \cdot \mathbf{p}_{i}}{\left(E_{f}+M_{f}\right)\left(E_{i}+M_{i}\right)}\right] \chi_{i},
\end{aligned}
$$

$$
\begin{aligned}
\bar{u}\left(\mathbf{p}_{f}, s_{f}\right) Q u\left(\mathbf{p}_{i}, s_{i}\right)= & \sqrt{\left(E_{f}+M_{f}\right)\left(E_{i}+M_{i}\right)} \chi_{f}^{\dagger} \\
& \times\left[\frac{1}{2}\left[\left(W_{f}-M_{f}\right)+\left(W_{i}-M_{i}\right)\right]\right. \\
& +\frac{1}{2}\left[\left(W_{f}+M_{f}\right)+\left(W_{i}+M_{i}\right)\right] \\
& \left.\times \frac{\boldsymbol{\sigma} \cdot \mathbf{p}_{f} \boldsymbol{\sigma} \cdot \mathbf{p}_{i}}{\left(E_{f}+M_{f}\right)\left(E_{i}+M_{i}\right)}\right] \chi_{i},
\end{aligned}
$$

with

$$
Q^{\mu}=\frac{1}{2}\left(q_{f}+q_{i}\right)^{\mu} .
$$

In Eq. (2.37) we used the shorthand notations $E_{f}=E\left(\mathbf{p}_{f}\right)$ etc. for the baryon variables. The meson variables were eliminated using $q^{0}=W-E$ etc. From the expressions in Eqs. (2.37), (2.5), and (2.29) we immediately obtain the relations between the amplitudes $F, G$ and $A, B$ :

$$
\begin{aligned}
F\left(\mathbf{p}_{f}, \mathbf{p}_{i}\right)= & \sqrt{\left(E_{f}+M_{f}\right)\left(E_{i}+M_{i}\right)}[A(s, t, u) \\
& \left.+\frac{W_{f}-M_{f}+W_{i}-M_{i}}{2} B(s, t, u)\right], \\
G\left(\mathbf{p}_{f}, \mathbf{p}_{i}\right)= & \sqrt{\left(E_{f}-M_{f}\right)\left(E_{i}-M_{i}\right)}[-A(s, t, u) \\
& \left.+\frac{W_{f}+M_{f}+W_{i}+M_{i}}{2} B(s, t, u)\right] .
\end{aligned}
$$

\section{THE PARTIAL WAVE EQUATION}

The NSC model is fitted to the partial wave analyses of the $\pi N$ and $K^{+} N$ scattering data; for this purpose the integral equation for the meson-baryon amplitude must be solved on the partial wave basis. This section deals with the transformation of the integral equation on the plane wave basis to the integral equation on the partial wave (LSJ) basis. From the unitarity of the scattering matrix, the relation between the partial wave amplitude and the partial wave phase shifts is derived. 


\section{A. Partial wave analysis ${ }^{1}$}

The states for the meson-baryon system are characterized by $J, L$, where $J$ is the total angular momentum and $L$ is the orbital angular momentum. The latter, for fixed $J$ value, can assume the values $L=J \mp 1 / 2$, since the spin of the baryons is $S=1 / 2$. Distinguishing between the partial waves with parity $P=(-)^{J-1 / 2}$ and $P=(-)^{J+1 / 2}$, using rotational invariance, we can write the potential matrix elements on the LSJ basis in the following way:

(i) $P=(-)^{L_{+}}, L_{+}=J-1 / 2$ :

$$
\left(q_{f} ; L^{\prime} J^{\prime} M^{\prime}|V| q_{i} ; L J M\right)=4 \pi V^{J, L_{+}}\left(L^{\prime}, L\right) \delta_{J^{\prime} J} \delta_{M^{\prime} M} \delta_{L^{\prime} L},
$$

(ii) $P=(-)^{L_{-}}, L_{-}=J+1 / 2$ :

$$
\left(q_{f} ; L^{\prime} J^{\prime} M^{\prime}|V| q_{i} ; L J M\right)=4 \pi V^{J, L_{-}}\left(L^{\prime}, L\right) \delta_{J^{\prime} J} \delta_{M^{\prime} M} \delta_{L^{\prime} L} .
$$

Because of parity conservation in strong interactions, the $L_{+}=J-1 / 2$ and the $L_{-}=J+1 / 2$ waves obviously are decoupled. So mixing between states with different angular momenta never occurs.

The spherical wave functions in momentum space with quantum numbers $J, M, L, S=1 / 2$ are

$$
\mathcal{Y}_{J L}^{M}(\hat{p}, s)=\sum_{m, \mu} C_{m \mu M}^{L \frac{1}{2} J} Y_{m}^{L}(\hat{p}) \chi_{\mu}^{\left(\frac{1}{2}\right)}(s),
$$

where $s$ is a spin variable for the baryons. For example, $s$ denotes the helicity of the baryon, or the projection of the spin along the normal $\hat{\mathbf{n}}$ to the scattering plane, or the projection of the spin along the $z$ axis. In this work, we will use the latter spin variable. Then, in Eq. (3.3) we have $\chi_{\mu}^{(1 / 2)}(s)=\delta_{s, \mu}$. The central and noncentral potential matrix elements on the LSJ basis are derived in detail in Appendix A1; the results are as follows:

(1) Central $P_{1}=1$ :

$$
\begin{aligned}
& \left(q_{f} ; L^{\prime} J^{\prime} M^{\prime}\left|F\left(\mathbf{q}_{f}, \mathbf{q}_{i}\right)\right| q_{i} ; L J M\right) \\
& \quad=4 \pi F_{L}\left(q_{f}, q_{i}\right) \delta_{L^{\prime} L} \delta_{J^{\prime}, J} \delta_{M^{\prime}, M},
\end{aligned}
$$

(2) NoncentralP $P_{2}^{\prime}=\left(\boldsymbol{\sigma} \cdot \hat{\mathbf{q}}_{f}\right)\left(\boldsymbol{\sigma} \cdot \hat{\mathbf{q}}_{i}\right)$ :

$$
\begin{aligned}
& \left(q_{f} ; L^{\prime} J^{\prime} M^{\prime}\left|G\left(\mathbf{q}_{f}, \mathbf{q}_{i}\right)\right| q_{i} ; L J M\right) \\
& \quad=4 \pi \sum_{L^{\prime \prime}} a_{L^{\prime}, L^{\prime \prime}} G_{L^{\prime \prime}}\left(q_{f}, q_{i}\right) a_{L^{\prime \prime}, L} \delta_{J^{\prime}, J} \delta_{M^{\prime}, M},
\end{aligned}
$$

where the partial wave projections $F_{L}$ and $G_{L}$ as well as the matrix $a_{L^{\prime}, L}$ are defined in Appendix A. The partial wave potentials $V^{J, L_{+}}$and $V^{J, L_{-}}$in Eqs. (3.1) and (3.2) can be expressed in terms of the partial wave expansions of $F$ and $G$. As expected from parity conservation, the partial wave potentials are diagonal in $L=(J \pm 1 / 2)$ space:

$$
V^{J, L_{ \pm}}=F_{L_{ \pm}}+G_{L_{ \pm} \pm 1} .
$$

\footnotetext{
${ }^{1}$ In this section we use the nonrelativistic normalization given by Eq. (2.33) of the two-particle states.
}

The partial wave potentials can also be expressed in terms of the partial wave projections of the central and spin-orbit potentials. The relation between $F$ and $G$ and the central and spin-orbit potentials is given by Eq. (2.30),

$$
F=V^{C}+\cos \theta V^{\mathrm{SO}}, \quad G=-V^{\mathrm{SO}} .
$$

The partial wave potentials in terms of the partial wave projections of the central and spin-orbit potentials becomes

$$
\begin{aligned}
V^{J, L_{p m}}= & \left\{\begin{array}{c}
V_{L_{+}}^{C}, \quad L_{+}=0, \\
V_{L_{ \pm}}^{C}+\frac{L_{ \pm}+1}{2 L_{ \pm}+1} V_{L_{ \pm}+1}^{\mathrm{SO}}
\end{array}\right. \\
& +\frac{L_{ \pm}}{2 L_{ \pm}+1} V_{L_{ \pm}-1}^{\mathrm{SO}}-V_{L_{ \pm}}^{\mathrm{SO}}, \quad L_{ \pm} \geqslant 1 .
\end{aligned}
$$

\section{B. Partial wave integral equations and the unitarity relations}

\section{Partial wave integral equations}

First, we write explicitly the integral equation (2.24) in terms of the plane wave states

$$
\begin{aligned}
\left(\mathbf{q}_{f},\right. & \left.s_{f}|T(\sqrt{s})| \mathbf{q}_{i}, s_{i}\right) \\
= & \left(\mathbf{q}_{f}, s_{f}|V(\sqrt{s})| \mathbf{q}_{i}, s\right)+\sum_{n} \int \frac{d^{3} k_{n}}{(2 \pi)^{3}}\left(\mathbf{q}_{f}, s_{f} \mid V(\sqrt{s})\right. \\
& \left.\times \mid \mathbf{k}_{n}, s_{n}\right) G_{0}\left(\mathbf{k}_{n}, \sqrt{s}\right)\left(\mathbf{k}_{n}, s_{n}|T(\sqrt{s})| \mathbf{q}_{i}, s_{i}\right),
\end{aligned}
$$

where, apart from spin space, the amplitude $T$, the Green's function $G_{0}$, and the potential $V$ are matrices in the two-particle channel space. The partial wave $T$ matrix for $L=L_{i}=L_{f}$ defined by

$$
T_{J, L}\left(q_{f}, q_{i} ; \sqrt{s}\right)=\left(q_{f} ; L J M|T(\sqrt{s})| q_{i} ; L J M\right),
$$

which is independent of $J_{z}=M$ owing to rotation invariance, is related to the $T$ matrix on the plane wave basis by

$$
\begin{aligned}
T_{J, L}\left(q_{f}, q_{i} ; \sqrt{s}\right)= & \sum_{s_{f}, s_{i}} \int \frac{d^{3} q_{f}^{\prime}}{(2 \pi)^{3}} \int \frac{d^{3} q_{i}^{\prime}}{(2 \pi)^{3}}\left(q_{f} ; L J M \mid \mathbf{q}_{f}^{\prime}, s_{f}\right) \\
& \times\left(\mathbf{q}_{f}^{\prime}, s_{f}|T(\sqrt{s})| \mathbf{q}_{i}^{\prime}, s_{i}\right)\left(\mathbf{q}_{i}^{\prime}, s_{i} \mid q_{i} ; L J M\right) .
\end{aligned}
$$

The integral equation for the partial wave amplitude now becomes

$$
\begin{aligned}
T_{J, L}\left(q_{f}, q_{i} ; \sqrt{s}\right)= & V_{J, L}\left(q_{f}, q_{i} ; \sqrt{s}\right)+\sum_{n} \int_{0}^{\infty} \frac{k_{n}^{2} d k_{n}}{(2 \pi)^{3}} \\
& \times V_{J, L}\left(q_{f}, q_{n} ; \sqrt{s}\right) G_{0}\left(k_{n}, \sqrt{s}\right) T_{J, L}\left(q_{n}, q_{i} ; \sqrt{s}\right) .
\end{aligned}
$$

\section{Partial wave unitarity relations and phase shifts}

From the unitarity of the $S$ matrix, $S^{\dagger} S=1$, the $M$ matrix in Eq. (2.2) satisfies the condition

$$
\begin{aligned}
2 \Im\langle f|M| i\rangle= & -(2 \pi)^{4} \sum_{n} \delta^{4}\left(P_{f}-P_{n}\right) \\
& \times\left\langle f\left|M^{\dagger}\right| n\right\rangle\langle n|M| i\rangle .
\end{aligned}
$$

In deriving Eq. (3.13) one factors out $\delta^{4}\left(P_{f}-P_{i}\right)$. The previous equation for the c.m. amplitudes can be written more 
explicitly (see, e.g., Eq. (II.1.14) of [23]) as

$$
\begin{aligned}
2 \Im & \left(\mathbf{q}_{f}, s_{f}|T| \mathbf{q}_{i}, s_{i}\right) \\
= & -\frac{1}{(2 \pi)^{2}} \sum_{n} \int \frac{d^{3} k_{n}}{4 E\left(\mathbf{k}_{n}\right) \mathcal{E}\left(\mathbf{k}_{n}\right)}\left(\mathbf{q}_{f}, s_{f}\left|T^{\dagger}\right| \mathbf{k}_{n}, s_{n}\right) \\
& \times \delta\left(\sqrt{s}-E\left(\mathbf{k}_{n}\right)-\mathcal{E}\left(\mathbf{k}_{n}\right)\right)\left(\mathbf{k}_{n}, s_{n}|T| \mathbf{q}_{i}, s_{i}\right),
\end{aligned}
$$

where the summation $\sum_{n}$ is over all intermediate two-particle channels coupled to the initial and final states. Here, $s_{i}, s_{f}$, and $s_{n}$ are the spin labels for the initial, final, and intermediate states, respectively. The momentum of the intermediate state $k_{n}$ is such that $E\left(\mathbf{k}_{n}\right)+\mathcal{E}\left(\mathbf{k}_{n}\right)=\sqrt{s}$. The unitarity relation for the partial wave amplitude then becomes

$$
2 \Im T_{J, L}\left(q_{f}, q_{i}\right)=-\sum_{n} \frac{q_{n}}{16 \pi^{2} \sqrt{s}} T_{J, L}^{\dagger}\left(q_{f}, q_{n}\right) T_{J, L}\left(q_{n}, q_{i}\right),
$$

where $q_{n}$ is the on-energy-shell momentum of the intermediate state. Introducing the partial wave amplitudes $F_{J, L}$ by the definition

$$
T_{J, L}=-32 \pi^{2} \sqrt{s} F_{J, L},
$$

we find the simple unitarity relation for these amplitudes:

$$
\Im F_{J, L}\left(q_{f}, q_{i}\right)=\sum_{c} q_{c} F_{J, L}\left(q_{f}, q_{c}\right)^{\dagger} F_{J, L}\left(q_{c}, q_{i}\right) .
$$

For the single-channel case $q_{c}=q_{i}=q_{f}=q$, phase shifts can be defined for the partial wave amplitude $F_{J, L}$ in the usual way:

$$
F_{J, L}=\frac{1}{q} \sin \delta_{J, L}(q) \exp \left(i \delta_{J, L}(q)\right) .
$$

The relation of $F_{J, L}$ with the partial wave $S$ matrix is

$$
S_{J, L}=e^{2 i \delta_{J, L}}=1+2 i q F_{J, L} .
$$

Now the expression for the differential cross section becomes

$$
\frac{d \sigma}{d \Omega}=|\tilde{f}|^{2}+|\tilde{g}|^{2}
$$

where the commonly used spin-nonflip and spin-flip amplitudes $\tilde{f}$ and $\tilde{g}$ are given by

$$
\begin{aligned}
& \tilde{f}=\frac{f}{8 \pi \sqrt{s}}=\sum_{L}\left[(L+1) F_{L+\frac{1}{2}, L}+L F_{L-\frac{1}{2}, L}\right] P_{L}(\cos \theta), \\
& \tilde{g}=\frac{g}{8 \pi \sqrt{s}}=\sum_{L}\left[F_{L+\frac{1}{2}, L}-F_{L-\frac{1}{2}, L}\right] \sin \theta \frac{d P_{L}(\cos \theta)}{d \cos \theta} .
\end{aligned}
$$

The expressions for the total cross section, which is found by integrating the differential cross section, and the polarization are

$$
\begin{aligned}
\sigma & =4 \pi \sum_{J} \frac{2 J+1}{2}\left(\left|F_{J, L_{+}}\right|^{2}+\left|F_{J, L_{-}}\right|^{2}\right), \\
P(\theta) & =\frac{2 \Im\left(\tilde{f} \tilde{g}^{*}\right)}{|\tilde{f}|^{2}+|\tilde{g}|^{2}} .
\end{aligned}
$$

\section{BARYON- AND MESON-EXCHANGE POTENTIALS}

The effective local interaction Hamiltonians that are used to calculate the one-hadron-exchange potentials are defined in this section. The Lorentz structure of the interaction is given and the $\mathrm{SU}_{f}(3)$ structure is reviewed in paper II, since we extend the NSC $\pi N$ model to the NSC $K^{+} N$ model. The amplitudes of the one-hadronexchange Feynman diagrams are given and a partial wave projection is made to find the partial wave potentials.

\section{A. The interaction Hamiltonians}

The potentials we use are obtained from the $t$-channel one-boson-exchange (OBE) and the $u$ - and $s$-channel baryon-exchange Feynman diagrams. In the $t$ channel we consider the exchange of vector, scalar and tensor mesons and in the $u$ and $s$ channel we consider the exchange of $J^{P}=1 / 2^{+}, 3 / 2^{+}$, and $1 / 2^{-}$baryons. In this work we also include Pomeron-exchange diagrams, where the physical nature of the Pomeron can be understood in the light of QCD as a two-gluon-exchange effect; see [25]. The contribution of the Pomeron will almost completely cancel the contribution of the isoscalar scalar meson $\sigma$.

The OBE Feynman diagrams for meson-baryon interactions contain a meson-baryon-baryon vertex and a meson-mesonmeson vertex. These vertices are determined by the effective local interaction Hamiltonian densities. The Lorentz structures of the local interaction densities for the meson-baryon-baryon (MBB) vertices we use are listed in the following:

(i) $J^{\mathrm{PC}}=0^{-+}$pseudoscalar mesons: For the pseudoscalar mesons we use the pseudovector interaction Hamiltonian

$$
\mathcal{H}_{P V}=\frac{f}{m_{\pi^{+}}} \bar{\psi}_{f} \gamma_{5} \gamma_{\mu} \psi_{i} \partial^{\mu} \phi_{P}
$$

which is scaled with the charged-pion mass to have a dimensionless pseudovector coupling constant.

(ii) $J^{\mathrm{PC}}=1^{-}$vector mesons: The interaction Hamiltonian is given in terms of the electric and magnetic interaction:

$$
\mathcal{H}_{V}=g_{V} \bar{\psi}_{f} \gamma_{\mu} \psi_{i} \phi_{V}^{\mu}+\frac{f_{V}}{4 \mathcal{M}} \bar{\psi}_{f} \sigma_{\mu \nu} \psi_{i}\left(\partial^{\mu} \phi_{V}^{v}-\partial^{\nu} \phi_{V}^{\mu}\right),
$$

where usually the proton mass is used for $\mathcal{M}$ to scale the magnetic part of the interaction Hamiltonian. The antisymmetric tensor operator used here is defined as $\sigma_{\mu \nu}=\frac{i}{2}\left[\gamma_{\mu}, \gamma_{\nu}\right]$

(iii) $J^{\mathrm{PC}}=0^{++}$scalar mesons: The interaction Hamiltonian is

$$
\mathcal{H}_{S}=g_{S} \bar{\psi}_{f} \psi_{i} \phi_{S}
$$

Since we include Pomeron exchange in the NSC model, scalar-meson exchange is canceled for the greater part; hence it is possible to satisfy the soft-pion theorem while including scalar-meson exchange. 
(iv) $J^{\mathrm{PC}}=2^{++}$tensor mesons: For the tensor mesons we use the interaction Hamiltonian

$$
\begin{aligned}
\mathcal{H}_{T}= & {\left[\frac{i}{4} \bar{\psi}_{f}\left(\gamma_{\mu} \stackrel{\leftrightarrow}{\partial_{\nu}}+\gamma_{\nu} \stackrel{\leftrightarrow}{\partial_{\mu}}\right) \psi_{i} F_{1}\right.} \\
& \left.-\frac{1}{4}\left(\bar{\psi}_{f} \quad \stackrel{\leftrightarrow}{\partial^{\mu}} \stackrel{\leftrightarrow}{\partial^{v}} \psi_{i}\right) F_{2}\right] \phi_{T}^{\mu \nu},
\end{aligned}
$$

where the coupling constants $F_{1}$ and $F_{2}$ are related to the dimensionless Pauli coupling constants by $G_{T, 1}=\mathcal{M} F_{1}$ and $G_{T, 2}=\mathcal{M}^{2} F_{2}$. By using the Gordon decomposition, the Pauli coupling constants are related to the Dirac coupling constants by $g_{T}=G_{T, 1}+G_{T, 2}$ and $f_{T}=-G_{T, 2}$.

(v) $J^{P}=3 / 2^{+}$resonance-baryon-pseudoscalar meson: The local interaction density for the $J^{P}=3 / 2^{+}$resonance -nucleon-pseudoscalar meson $\left(Y^{*} N P\right)$ interaction is

$$
\mathcal{H}_{Y^{*} N P}=-i \frac{f^{*}}{m_{\pi^{+}}} \bar{\psi}_{N} \psi_{Y^{*}, \mu} \partial^{\mu} \phi_{P},
$$

where the charged-pion mass makes the coupling dimensionless. We use the Rarita-Schwinger formalism for the spin-3/2 resonances (see, e.g., Refs. [19,26]).

(vi) $J^{P}=1 / 2^{-}$resonance-baryon-pseudoscalar meson: The local interaction Hamiltonian for the $J^{P}=1 / 2^{-}$ resonance-nucleon-pseudoscalar meson $(R N P)$ interaction is

$$
\mathcal{H}_{R N P}=\frac{f^{*(v)}}{m_{\pi^{+}}} \bar{\psi}_{N} \gamma_{\mu} \psi_{R} \partial^{\mu} \phi_{P},
$$

where $\psi_{R}$ denotes the $J^{P}=1 / 2^{-}$resonance, which has opposite parity to the nucleon. The $J^{P}=1 / 2^{-}$ resonances we consider in this work are the $S_{11}(1555)$ in the $\pi N$ system and the $\Lambda(1405)$ in the $K N$ system.

Here $\phi$ denotes the pseudoscalar-, vector-, scalar-, and tensor-meson fields, respectively, and $\psi$ denotes the baryon fields. The Pomeron-baryon-baryon interaction density we use has the same Lorentz structure as the scalar mesons.

We note that, by making use of the Dirac equation $\left(\gamma^{\mu} \partial_{\mu}+M\right) \psi=0$, the pseudovector interaction Hamiltonian density in Eq. (4.1) is "equivalent" to the pseudoscalar density $\mathcal{H}_{P S}=\operatorname{ig} \bar{\psi}_{f} \gamma_{5} \psi_{i} \phi_{P}$ for on-mass-shell particles. The coupling constants are then related according to $g /\left(M_{B_{f}}+\right.$ $\left.M_{B_{i}}\right)=f / m_{\pi^{+}}$. Analogously, we find that the vector coupling Hamiltonian density in Eq. (4.6) is "equivalent" to the scalar density $\mathcal{H}_{S}=i g^{*(s)} \bar{\psi}_{N} \psi_{R} \phi_{P}$ for on-mass-shell particles. The coupling constants are in this case related according to $g^{*(s)} /\left(M_{N}-M_{R}\right)=f^{*(v)} / m_{\pi^{+}}$.

The Lorentz structure of the local interaction density for triple-meson (MMM) vertices is schematically given in the following and are discussed in more detail in paper II:

(i) $J^{\mathrm{PC}}=1^{-}$vector mesons:

$$
\mathcal{H}_{P P V}=g_{P P V} \phi_{V}^{\mu}\left(\phi_{P} \stackrel{\leftrightarrow}{\partial}_{\mu} \phi_{P}\right)
$$

(ii) $J^{\mathrm{PC}}=0^{++}$scalar mesons:

$$
\mathcal{H}_{P P S}=g_{P P S} \phi_{S}\left(\phi_{P} \phi_{P}\right)
$$

(iii) $J^{\mathrm{PC}}=2^{++}$tensor mesons:

$$
\mathcal{H}_{P P T}=\frac{2 g_{P P T}}{m_{\pi^{+}}} \phi_{T}^{\mu v}\left(\partial_{\mu} \phi_{P}\right)\left(\partial_{\nu} \phi_{P}\right)
$$

Concerning the flavor structure of the interaction densities, we assume that the coupling constants are related via $\mathrm{SU}_{f}(3)$ symmetry, as outlined in paper II, here the relevant isoscalar and isospin factors are given. However, the potentials will break $\mathrm{SU}_{f}(3)$ symmetry dynamically, since we use the physical masses of the particles.

\section{B. The relativistic invariant amplitudes}

Using the previously defined interaction Hamiltonians, we give, except for the isospin and isoscalar factors, the contributions to the relativistic invariant amplitudes $A(s, t, u)$ and $B(s, t, u)$ in Eq. (2.5) for the elastic (e.g., $\pi N$ and $\left.K^{+} N\right)$ channels, that is, $M_{i}=M_{f} \equiv M, m_{i}=m_{f}=m$, where $M_{f}$ and $M_{I}$ are the final and initial baryon masses and $m_{f}$ and $m_{i}$ are the final and initial pseudoscalar-meson masses, respectively. Amplitudes for the general mass case are listed in Appendix B.

\section{Baryon-exchange amplitudes}

For $J^{P}=1 / 2^{+}$baryon exchange the relativistic amplitudes are

$$
\begin{aligned}
A_{p s}(s, t, u)= & -\frac{g_{14} g_{23}}{u-M_{B}^{2}+i \epsilon}\left[M_{B}-M\right], \\
B_{p s}(s, t, u)= & -\frac{g_{14} g_{23}}{u-M_{B}^{2}+i \epsilon}, \\
A_{p v}(s, t, u)= & -\frac{f_{14} f_{23} / m_{\pi^{+}}^{2}}{u-M_{B}^{2}+i \epsilon}\left[u\left(M+M_{B}\right)\right. \\
& \left.-M^{3}-M^{2} M_{B}\right], \\
B_{p v}(s, t, u)= & -\frac{f_{14} f_{23} / m_{\pi^{+}}^{2}}{u-M_{B}^{2}+i \epsilon}\left[u+2 M M_{B}+M^{2}\right],
\end{aligned}
$$

for pseudoscalar $(p s)$ and pseudovector $(p v)$ coupling, respectively; $M_{B}$ is the mass of the exchanged baryon. The $J^{P}=1 / 2^{+}$baryon direct pole gives rise to the relativistic amplitudes

$$
\begin{aligned}
A_{p s}(s, t, u)= & -\frac{g_{12} g_{34}}{s-M_{B}^{2}+i \epsilon}\left[M_{B}-M\right], \\
B_{p s}(s, t, u)= & \frac{g_{12} g_{34}}{s-M_{B}^{2}+i \epsilon}, \\
A_{p v}(s, t, u)= & -\frac{f_{12} f_{34} / m_{\pi^{+}}^{2}}{s-M_{B}^{2}+i \epsilon}\left[s\left(M+M_{B}\right)\right. \\
& \left.-M^{3}-M^{2} M_{B}\right], \\
B_{p v}(s, t, u)= & \frac{f_{12} f_{34} / m_{\pi^{+}}^{2}}{s-M_{B}^{2}+i \epsilon}\left[s+2 M M_{B}+M^{2}\right],
\end{aligned}
$$


for pseudoscalar and pseudovector coupling, respectively. For $J^{P}=1 / 2^{-}$baryon exchange the relativistic amplitudes are

$$
\begin{aligned}
A_{s}(s, t, u)= & \frac{g_{14}^{*(s)} g_{23}^{*(s)}}{u-M_{B}^{2}+i \epsilon}\left[M_{B}+M\right], \\
B_{s}(s, t, u)= & -\frac{g_{14}^{*(s)} g_{23}^{*(s)}}{u-M_{B}^{2}+i \epsilon}, \\
A_{v}(s, t, u)= & \frac{f_{14}^{*(v)} f_{23}^{*(v)} / m_{\pi^{+}}^{2}}{u-M_{B}^{2}+i \epsilon}\left[u\left(-M+M_{B}\right)\right. \\
& \left.+M^{3}-M^{2} M_{B}\right], \\
B_{v}(s, t, u)= & \frac{f_{14}^{*(v)} f_{23}^{*(v)} / m_{\pi^{+}}^{2}}{u-M_{B}^{2}+i \epsilon}\left[-u+2 M M_{B}-M^{2}\right],
\end{aligned}
$$

for scalar $(s)$ and vector $(v)$ coupling, respectively; $M_{B}$ is the mass of the exchanged baryon. The $J^{P}=1 / 2^{-}$baryon direct pole gives rise to the relativistic amplitudes

$$
\begin{aligned}
A_{s}(s, t, u)= & \frac{g_{12}^{*(s)} g_{34}^{*(s)}}{s-M_{B}^{2}+i \epsilon}\left[M_{B}+M\right], \\
B_{s}(s, t, u)= & \frac{g_{12}^{*(s)} g_{34}^{*(s)}}{s-M_{B}^{2}+i \epsilon}, \\
A_{v}(s, t, u)= & \frac{f_{12}^{*(v)} f_{34}^{*(v)} / m_{\pi^{+}}^{2}}{s-M_{B}^{2}+i \epsilon}\left[s\left(-M+M_{B}\right)\right. \\
& \left.+M^{3}-M^{2} M_{B}\right], \\
B_{v}(s, t, u)= & -\frac{f_{12}^{*(v)} f_{34}^{*(v)} / m_{\pi^{+}}^{2}}{s-M_{B}^{2}+i \epsilon}\left[-s+2 M M_{B}-M^{2}\right],
\end{aligned}
$$

for scalar and vector coupling, respectively. The $J^{P}=3 / 2^{+}$ resonance-exchange relativistic amplitudes are more complicated:

$$
\begin{aligned}
A_{Y^{*}}(s, t, u)= & \frac{f_{14}^{*} f_{23}^{*} / m_{\pi^{+}}^{2}}{u-M_{Y^{*}}^{2}+i \epsilon}\left[\frac{\left[t-2 m^{2}\right]}{2}\left(M+M_{Y^{*}}\right)\right. \\
& +\frac{M_{Y^{*}}}{3}\left[u-M^{2}\right]+\frac{1}{6 M_{Y^{*}}}\left[-u^{2}+2 M u\right. \\
& \left.\times\left(M+M_{Y^{*}}\right)-2 M^{3} M_{Y^{*}}-M^{4}+m^{4}\right] \\
& \left.+\frac{1}{6 M_{Y^{*}}^{2}}\left[M^{2}-m^{2}-u\right]^{2}\left(M+M_{Y^{*}}\right)\right], \\
B_{Y^{*}}(s, t, u)= & \frac{f_{14}^{*} f_{23}^{*} / m_{\pi^{+}}^{2}}{u-M_{Y^{*}}^{2}+i \epsilon}\left[-\frac{\left[t-2 m^{2}\right]}{2}+\frac{1}{3 M_{Y^{*}}}\right. \\
& \times\left[\left(M+M_{Y^{*}}\right)\left(2 M M_{Y^{*}}-m^{2}\right)-u M+M^{3}\right] \\
& \left.-\frac{1}{6 M_{Y^{*}}^{2}}\left[u-M^{2}+m^{2}\right]^{2}\right],
\end{aligned}
$$

where $M_{Y^{*}}$ is the mass of the exchanged resonance. The $J^{P}=3 / 2^{+}$resonance direct pole gives rise to the relativistic amplitudes

$$
\begin{aligned}
& A_{Y^{*}}(s, t, u)=\frac{f_{12}^{*} f_{34}^{*} / m_{\pi^{+}}^{2}}{s-M_{Y^{*}}^{2}+i \epsilon}\left[\frac{\left[t-2 m^{2}\right]}{2}\left(M+M_{Y^{*}}\right)\right. \\
& +\frac{M_{Y^{*}}}{3}\left[s-M^{2}\right]+\frac{1}{6 M_{Y^{*}}}\left[-s^{2}+2 M s\right. \\
& \left.\times\left(M+M_{Y^{*}}\right)-2 M^{3} M_{Y^{*}}-M^{4}+m^{4}\right] \\
& \left.+\frac{1}{6 M_{Y^{*}}^{2}}\left[M^{2}-m^{2}-s\right]^{2}\left(M+M_{Y^{*}}\right)\right], \\
& B_{Y^{*}}(s, t, u)=-\frac{f_{12}^{*} f_{34}^{*} / m_{\pi^{+}}^{2}}{s-M_{Y^{*}}^{2}+i \epsilon}\left[-\frac{\left[t-2 m^{2}\right]}{2}+\frac{1}{3 M_{Y^{*}}}\right. \\
& \times\left[\left(M+M_{Y^{*}}\right)\left(2 M M_{Y^{*}}-m^{2}\right)-s M+M^{3}\right] \\
& \left.-\frac{1}{6 M_{Y^{*}}^{2}}\left[s-M^{2}+m^{2}\right]^{2}\right] \text {. }
\end{aligned}
$$

\section{Meson- and Pomeron-exchange amplitudes}

The relativistic amplitudes for the $t$-channel Pomeron exchange, scalar-meson exchange, vector-meson exchange, and tensor-meson exchange are

$$
\begin{aligned}
A_{P}(s, t, u)= & \frac{g_{P P P} g_{P}}{\mathcal{M}}, \quad B_{P}(s, t, u)=0, \\
A_{S}(s, t, u)= & \frac{g_{P P S} g_{S}}{t-m_{S}^{2}+i \epsilon}, \quad B_{S}(s, t, u)=0, \\
A_{V}(s, t, u)= & \frac{g_{P P V}}{t-m_{V}^{2}+i \epsilon} \frac{f_{V}}{2 \mathcal{M}}[s-u], \\
B_{V}(s, t, u)= & -2 \frac{g_{P P V}}{t-m_{V}^{2}+i \epsilon}\left[g_{V}+\frac{M}{\mathcal{M}} f_{V}\right], \\
A_{T}(s, t, u)= & \frac{g_{P P T} / m_{\pi^{+}}}{t-m_{T}^{2}+i \epsilon}\left[\frac{1}{4}(s-u)^{2} F_{2}-\frac{1}{6}\left[4 m^{2}-t\right]\right. \\
& \left.\times\left[2 M F_{1}+\frac{1}{2}\left(4 M^{2}-t\right) F_{2}\right]\right], \\
B_{T}(s, t, u)= & \frac{g_{P P T} / m_{\pi^{+}}}{t-m_{T}^{2}+i \epsilon}[s-u] F_{1},
\end{aligned}
$$

where $m_{S}, m_{V}$, and $m_{T}$ are the masses of the exchanged scalar meson, vector meson, and tensor meson, respectively.

Here we notice that for the meson-exchange and Pomeronexchange amplitudes an extra factor of 2 must be added to the amplitudes if both the initial and final state contain a $\pi$ or $\eta$; this is not the case for any other combination of pseudoscalar mesons in the initial and final states. For elastic $\pi N$ scattering, for example, an extra factor of 2 is added to the $\rho$ exchange, Pomeron-exchange, and $\sigma$ exchange amplitudes.

\section{Partial wave potentials}

As discussed in Sec. III we solve the integral equation for the $T$ matrix on the partial wave basis, Eq. (3.12). And in paper II we fit the NSC model to the $\pi N$ partial wave 
analysis [1] and the $K^{+} N$ partial wave analysis [5]. For this purpose we need to calculate the partial wave projection of the potentials, Eq. (3.6). In our approximation, the potentials are given by the invariant amplitudes $A$ and $B$, Eqs. (4.10)(4.16), of the one-meson-exchange and one-baryon-exchange Feynman diagrams.

Until this point, we did not mention the need for form factors to regulate the high-energy behavior (i.e., the short distance behavior) of the potentials, but in fact the kernel of the integral equation without form factors does not satisfy the Fredholm condition, $\iint d p d k|K(p, k)|^{2}<\infty$, in general. Furthermore we have derived our one-meson-exchange and one-baryonexchange potentials from QFT, which is in principle only valid for point particles, whereas mesons and baryons have internal structure. Therefore we need to take into account the extended size of the mesons and baryons by means of a form factor. Since the ground-state wave functions of the quarks are Gaussian in many quark models, form factors of the Gaussian type are used in the NSC model. For $t$-channel exchanges we multiply the potentials by the form factor

$$
F(\Lambda)=e^{-\left(\mathbf{p}_{f}-\mathbf{p}_{i}\right)^{2} / \Lambda^{2}},
$$

where $\mathbf{p}_{i}$ and $\mathbf{p}_{f}$ are the c.m. three-momenta for the initial and final states, respectively; that is, at both vertices we have used the difference between the final and initial three-momenta. $\Lambda$ is a cutoff mass, which will be determined in the fit to the experimental phases.

For $u$ - and $s$-channel exchanges, the difference between the final and initial three-momenta of the baryon is used, giving the form factor

$$
F(\Lambda)=e^{-\left(\mathbf{p}_{f}^{2}+\mathbf{p}_{i}^{2}\right) / 2 \Lambda^{2}} .
$$

This form factor obviously does not depend on the scattering angle $\theta$, which simplifies the partial wave projection. For the $u$ and $t$ channel we rewrite the denominators of the potentials in the form

$$
\begin{aligned}
\frac{1}{t-m^{2}} & =\frac{-1}{2 p_{f} p_{i}} \frac{1}{z_{t}-x}, \\
\frac{1}{u-m^{2}} & =\frac{-1}{2 p_{f} p_{i}} \frac{1}{z_{u}+x},
\end{aligned}
$$

where $x=\cos (\theta)$ and $\theta$ is the angle between the final and initial three-momenta $\mathbf{p}_{f}$ and $\mathbf{p}_{i}$. Here we have defined the $z_{t}$ and $z_{u}$ factors as

$$
\begin{aligned}
& z_{t}=\frac{1}{2 p_{f} p_{i}}\left[m^{2}+p_{f}^{2}+p_{i}^{2}-\frac{1}{4}\left[E_{i}-E_{f}-\omega_{i}+\omega_{f}\right]^{2}\right], \\
& z_{u}=\frac{1}{2 p_{f} p_{i}}\left[m^{2}+p_{f}^{2}+p_{i}^{2}-\frac{1}{4}\left[E_{i}+E_{f}-\omega_{i}-\omega_{f}\right]^{2}\right],
\end{aligned}
$$

where $E_{f, i}$ are the baryon energies, $\omega_{f, i}$ are the meson energies, and $m$ is the mass of the exchanged particle. For positive and real momenta (i.e., for open channels), we have $z>1$. Now it is clear that the potentials $V^{(\alpha)}$ of Eq. (2.31), where $\alpha$ stands for central or spin orbit, can be expanded in $x$ as $^{2}$

$$
\begin{aligned}
V^{(\alpha)}\left(\mathbf{p}_{f}, \mathbf{p}_{i}\right) & =\frac{1}{2 p_{f} p_{i}}\left[X^{(\alpha)}+x Y^{(\alpha)}+x^{2} Z^{(\alpha)}\right] \frac{F\left(\Lambda_{t}\right)}{z_{t}-x}, \\
V^{(\alpha)}\left(\mathbf{p}_{f}, \mathbf{p}_{i}\right) & =\frac{1}{2 p_{f} p_{i}}\left[X^{(\alpha)}+x Y^{(\alpha)}+x^{2} Z^{(\alpha)}\right] \frac{F\left(\Lambda_{u}\right)}{z_{u}+x}, \\
V^{(\alpha)}\left(\mathbf{p}_{f}, \mathbf{p}_{i}\right) & =\left[X^{(\alpha)}+x Y^{(\alpha)}\right] \frac{F\left(\Lambda_{s}\right)}{s-M_{B}^{2}},
\end{aligned}
$$

for $t$-, $u$ - and $s$-channel exchanges, respectively, for all particles that are exchanged. The coefficients $X^{(\alpha)}, Y^{(\alpha)}$ and $Z^{(\alpha)}$ can be found easily by writing out the $x$ dependence of the invariant amplitudes $A$ and $B$; these are listed in Appendix $C$ for each type of exchange.

The partial wave potentials $V_{L}^{(\alpha)}$ are found by inverting the partial wave expansion Eq. (A2), giving

$$
V_{L}^{(\alpha)}\left(p_{f}, p_{i}\right)=\frac{1}{2} \int_{-1}^{1} d x P_{L}(x) V^{(\alpha)}\left(\mathbf{p}_{f}, \mathbf{p}_{i}\right) .
$$

The partial wave potentials now take the form

$$
\begin{aligned}
V_{L}^{(\alpha)}\left(p_{f}, p_{i}\right)= & {\left[X^{(\alpha)} \frac{F\left(\Lambda_{s}\right)}{s-M_{B}^{2}} \delta_{L, 0}+Y^{(\alpha)} \frac{F\left(\Lambda_{s}\right)}{s-M_{B}^{2}} \frac{\delta_{L, 1}}{3}\right] } \\
V_{L}^{(\alpha)}\left(p_{f}, p_{i}\right)= & \frac{1}{2 p_{f} p_{i}}\left[\left(X^{(\alpha)}+z_{t} Y^{(\alpha)}+z_{t}^{2} Z^{(\alpha)}\right)\right. \\
& \times U_{L}\left(\Lambda_{t}, z_{t}\right)-\left(Y^{(\alpha)}+z_{t} Z^{(\alpha)}\right) R_{L}\left(\Lambda_{t}, z_{t}\right) \\
& \left.-Z^{(\alpha)} S_{L}\left(\Lambda_{t}, z_{t}\right)\right] \\
V_{L}^{(\alpha)}\left(p_{f}, p_{i}\right)= & \frac{(-1)^{L}}{2 p_{f} p_{i}}\left[\left(X^{(\alpha)}-z_{u} Y^{(\alpha)}+z_{u}^{2} Z^{(\alpha)}\right)\right. \\
& \times U_{L}\left(\Lambda_{u}, z_{u}\right)-\left(-Y^{(\alpha)}+z_{u} Z^{(\alpha)}\right) R_{L}\left(\Lambda_{u}, z_{u}\right) \\
& \left.-Z^{(\alpha)} S_{L}\left(\Lambda_{u}, z_{u}\right)\right],
\end{aligned}
$$

for $s-, t$, and $u$-channel exchanges, respectively. We have defined the basic partial wave projections $U_{L}, R_{L}, S_{L}$, and $T_{L}$ in terms of the Legendre polynomials $P_{L}(x)$ and the form factors

$$
\begin{aligned}
& U_{L}(\Lambda, z)=\frac{1}{2} \int_{-1}^{1} d x \frac{P_{L}(x) F(\Lambda)}{z-x}, \\
& R_{L}(\Lambda, z)=\frac{1}{2} \int_{-1}^{1} d x P_{L}(x) F(\Lambda), \\
& S_{L}(\Lambda, z)=\frac{1}{2} \int_{-1}^{1} d x P_{L}(x) x F(\Lambda), \\
& T_{L}(\Lambda, z)=\frac{1}{2} \int_{-1}^{1} d x P_{L}(x) x^{2} F(\Lambda) .
\end{aligned}
$$

\footnotetext{
${ }^{2}$ For more complicated exchanges (e.g., $J^{\mathrm{PC}}=3 / 2^{+}$resonance), the expansions of the potentials have an additional term of higher order in $x$, for the $t$ - and $u$-channel $x^{3} U^{(\alpha)}$, and for the $s$-channel $x^{2} Z^{(\alpha)}$.

We notice that a similar expansion for $F$ and $G$ instead of $V^{(C)}$ and $V^{(\mathrm{SO})}$ would be a little simpler. However, we will use the central and spin-orbit potentials in light of a momentum space version of the NSC model.
} 
The factor $(-)^{L}$ appearing in the $u$-channel partial wave potentials, which is the result of changing the integration variable $x \rightarrow-x$ in the Legendre polynomial, is typical for exchange forces. In this way it can be seen that the total partial wave potential is a linear combination of a direct and an exchange potential, $V^{( \pm)}=V_{d} \pm V_{e}$, and the corresponding $T$ matrix is $T^{( \pm)}=T_{d} \pm T_{e}$. The amplitudes $T_{d}$ and $T_{e}$ do not satisfy an integral equation, but the two linear combinations $T^{( \pm)}=T_{d} \pm T_{e}$ do.

We notice that if the form factor does not depend on $x$ (in case of $u$-channel potentials) or if we consider the limit $\Lambda \rightarrow \infty$ [i.e., $F(\Lambda) \rightarrow 1$ ], the basic partial wave projections defined in Eq. (4.24) are proportional to the simple functions

$$
\begin{aligned}
U_{L}(\Lambda, z) & \propto Q_{L}(z), \\
R_{L}(\Lambda, z) & \propto \delta_{L, 0}, \\
S_{L}(\Lambda, z) & \propto \frac{1}{3} \delta_{L, 1}, \\
T_{L}(\Lambda, z) & \propto \frac{1}{3} \delta_{L, 0}+\frac{2}{15} \delta_{L, 2},
\end{aligned}
$$

where $Q_{L}(z)$ is the Legendre function of the second kind, which is an analytic function of its argument except for a cut on the real axis running from -1 to 1 , as is clear from Eq. (4.24). In view of Eq. (4.20), the cut is entered only for on-energyshell potentials below threshold, but we always calculate the on-energy-shell potentials above threshold, so we will never reach the cut.

In the NSC $\pi N$ model we will include $s$-channel baryonexchange diagrams, which are in principle separable diagrams, having the form

$$
V\left(p_{f}, p_{i}\right)=\frac{\Gamma\left(p_{f}\right) \Gamma\left(p_{i}\right)}{\sqrt{s} \pm M_{0}} .
$$

Writing out the partial wave potential for the $\Delta$ pole ( $P_{33}$ wave) explicitly, using Eq. (3.6) and Eq. (4.23),

$$
\begin{aligned}
V_{33}= & \frac{f_{\pi N \Delta}^{2}}{m_{\pi^{+}}^{2}} \frac{1}{3} \sqrt{\left(E_{i}+M_{i}\right)\left(E_{f}+M_{f}\right)} \\
& \times p_{f} p_{i} \frac{1}{\sqrt{s}-M_{0}},
\end{aligned}
$$

we see that this potential is of the separable kind indeed.

We need to be careful in including the $s$-channel diagrams in a model that has been renormalized, that is, in which (renormalized) physical coupling constants and masses are used. It is not possible to simply add the $s$-channel diagrams to the other ones, because iterations of $s$-channel diagrams will give contributions to the vertex and self-energy. The way these diagrams are included in the NSC model is described in paper II; here we show that bare masses and coupling constants should be used in the $s$-channel diagrams and that these bare parameters are determined by requiring that (i) the $T$ matrix has a pole at the physical mass $\sqrt{s}=M_{e}$ and (ii) the residue at the pole is given by the physical coupling constant.

\section{SUMMARY}

Analogous to the Nijmegen soft-core one-boson-exchange $N N$ and $Y N$ models, we have derived the NSC model for the interaction between pseudoscalar mesons and baryons $\left(\pi N, K^{+} N\right.$, etc.).

For the general mass case the meson-baryon potentials in the context of a relativistic two-particle equation, the BetheSalpeter equation, are defined in Sec. II. The potentials consist of one-meson-exchange and one-baryon-exchange Feynman diagrams. The Bethe-Salpeter equation is approximated by assuming dynamical pair suppression, hence by neglecting the propagation of negative-energy states, and by integrating over the time variable, giving a three-dimensional integral equation for the scattering amplitude, which is a generalization of the Lippmann-Schwinger equation.

A transformation of this equation on the plane wave basis to the partial wave (LSJ) basis is described in Sec. III. A onedimensional integral equation for the partial wave scattering amplitude is derived, which is decoupled for each partial wave, because of parity conservation in strong interactions.

In Sec. IV the interaction Hamiltonians are given and the resulting one-baryon-exchange and one-meson-exchange invariant amplitudes have been derived; these amplitudes define the partial wave potentials used in the calculations.

\section{ACKNOWLEDGMENTS}

The authors would like to thank Professor J. J. de Swart and Professor R. G. E. Timmermans for stimulating discussions.

\section{APPENDIX A: MATRIX ELEMENTS ON THE LSJ BASIS}

\section{Partial wave amplitudes}

Here we derive the central and noncentral potential matrix elements on the LSJ basis in Eqs. (3.4) and (3.5).

(i) Central $P_{1}=1$ :

$$
\begin{aligned}
& \left(q_{f} ; L^{\prime} J^{\prime} M^{\prime}\left|F\left(\mathbf{q}_{f}, \mathbf{q}_{i}\right)\right| q_{i} ; L J M\right) \\
& =\sum_{s_{f}, s_{i}} \int \frac{d^{3} q_{f}^{\prime}}{(2 \pi)^{3}} \int \frac{d^{3} q_{i}^{\prime}}{(2 \pi)^{3}}\left(q_{f} ; L^{\prime} J^{\prime} M^{\prime} \mid \mathbf{q}_{f}^{\prime}, s_{f}\right) \\
& \quad \times\left(\mathbf{q}_{f}^{\prime}, s_{f}\left|F_{\mathrm{op}}\right| \mathbf{q}_{i}^{\prime}, s_{i}\right)\left(\mathbf{q}_{i}^{\prime}, s_{i} \mid q_{i}, L J M\right)
\end{aligned}
$$

We now use the matrix elements

$$
\begin{aligned}
\left(\mathbf{q}_{f}^{\prime}, s_{f}\left|F_{\mathrm{op}}\right| \mathbf{q}_{i}^{\prime}, s_{i}\right)= & F\left(\mathbf{q}_{f}^{\prime}, \mathbf{q}_{i}^{\prime}\right) \delta_{s_{f}, s_{i}} \\
= & 4 \pi \sum_{l=0}^{\infty} F_{l}\left(q_{f}^{\prime}, q_{i}^{\prime}\right) \\
& \times \sum_{n=-l}^{l} Y_{n}^{l}\left(\hat{\mathbf{q}}_{f}^{\prime}\right) Y_{n}^{l}\left(\hat{\mathbf{q}}_{i}^{\prime}\right)^{*} \delta_{s_{f}, s_{i}}, \\
\left(\mathbf{q}^{\prime}, s \mid q, L J M\right)= & (2 \pi)^{3} \frac{\delta\left(q^{\prime}-q\right)}{q^{2}} \mathcal{Y}_{J L}^{M}\left(\hat{\mathbf{q}}^{\prime}, s\right) .
\end{aligned}
$$

Then, substituting Eq. (A2) into Eq. (A1) and performing the momentum and angular integrals and summations, we find

$$
\begin{aligned}
& \left(q_{f} ; L^{\prime} J^{\prime} M^{\prime}\left|F\left(\mathbf{q}_{f}, \mathbf{q}_{i}\right)\right| q_{i} ; L J M\right) \\
& \quad=4 \pi F_{L}\left(q_{f}, q_{i}\right) \delta_{L^{\prime}, L} \delta_{J^{\prime}, J} \delta_{M^{\prime}, M} .
\end{aligned}
$$


(ii) Noncentral $P_{2}^{\prime}=\left(\sigma \cdot \hat{\mathbf{q}}_{f}\right)\left(\sigma \cdot \hat{\mathbf{q}}_{i}\right)$ :

$$
\begin{aligned}
& \left(q_{f} ; L^{\prime} J^{\prime} M^{\prime}\left|G\left(\mathbf{q}_{f}, \mathbf{q}_{i}\right)\right| q_{i} ; L J M\right) \\
& =\sum_{s_{f}, s_{i}} \int \frac{d^{3} q_{f}^{\prime}}{(2 \pi)^{3}} \int \frac{d^{3} q_{i}^{\prime}}{(2 \pi)^{3}}\left(q_{f}^{\prime} ; L^{\prime} J^{\prime} M^{\prime} \mid \mathbf{q}_{f}^{\prime}, s_{f}\right) \\
& \quad \times\left(\mathbf{q}_{f}^{\prime}, s_{f}\left|G_{\mathrm{op}}\right| \mathbf{q}_{i}^{\prime}, s_{i}\right)\left(\mathbf{q}_{i}^{\prime}, s_{i} \mid q_{i}, L J M\right),
\end{aligned}
$$

where, analogously to Eq. (A2),

$$
\begin{gathered}
\left(\mathbf{q}_{f}^{\prime}, s_{f}\left|G_{\mathrm{op}}\right| \mathbf{q}_{i}^{\prime}, s_{i}\right) \\
=4 \pi \sum_{l=0}^{\infty} G_{l}\left(q_{f}^{\prime}, q_{i}^{\prime}\right) \sum_{n=-l}^{l} Y_{n}^{l}\left(\hat{\mathbf{q}}_{f}^{\prime}\right) Y_{n}^{l}\left(\hat{\mathbf{q}}_{i}^{\prime}\right)^{*} \\
\cdot \sum_{s}\left(s_{f}\left|\left(\sigma \cdot \hat{\mathbf{q}}_{f}^{\prime}\right)\right| s\right)\left(s\left|\left(\sigma \cdot \hat{\mathbf{q}}_{i}^{\prime}\right)\right| s_{i}\right) .
\end{gathered}
$$

Using Eq. (A2) and substituting Eq. (A5) into Eq. (A4), and performing the momentum integrals, we find

$$
\begin{aligned}
& \left(q_{f} ; L^{\prime} J^{\prime} M^{\prime}\left|G_{\mathrm{op}}\right| q_{i} ; L J M\right) \\
& =4 \pi \sum_{s_{f}, s_{i}, s} \sum_{l} G_{l}\left(q_{f}, q_{i}\right) \sum_{n=-l}^{l} \int d \hat{\Omega}_{q_{f}} \mathcal{Y}_{J^{\prime} L^{\prime}}^{M^{\prime}}\left(\hat{\mathbf{q}}_{f}, s_{f}\right)^{*} \\
& \quad \times\left(s_{f}\left|\left(\boldsymbol{\sigma} \cdot \hat{\mathbf{q}}_{f}\right)\right| s\right) Y_{n}^{l}\left(\hat{\mathbf{q}}_{f}\right) \int d \hat{\Omega}_{q_{i}} Y_{n}^{l}\left(\hat{\mathbf{q}}_{i}\right)^{*} \\
& \quad \times\left(s\left|\left(\sigma \cdot \hat{\mathbf{q}}_{i}\right)\right| s_{i}\right) \mathcal{Y}_{J L}^{M}\left(\hat{\mathbf{q}}_{i}, s_{i}\right) .
\end{aligned}
$$

In the two-dimensional $L=J \mp 1 / 2$ space, the $(\sigma \cdot \hat{\mathbf{q}})$ operator has the matrix elements (see Appendix A2),

$$
\begin{aligned}
& \sum_{s^{\prime}}\left(s|(\boldsymbol{\sigma} \cdot \hat{\mathbf{q}})| s^{\prime}\right) \mathcal{Y}_{J L}^{M}\left(\hat{\mathbf{q}}, s^{\prime}\right) \\
& \quad=\sum_{L^{\prime}} \mathcal{Y}_{J L^{\prime}}^{M}(\hat{\mathbf{q}}, s) a_{L^{\prime}, L}, \text { where } a_{L^{\prime}, L}=\left(\begin{array}{cc}
0 & -1 \\
-1 & 0
\end{array}\right) .
\end{aligned}
$$

The angular integrals in Eq. (A6) can now be performed easily. Then, the result for the noncentral amplitude is

$$
\begin{aligned}
& \left(q_{f} ; L^{\prime} J^{\prime} M^{\prime}|G| q_{i} ; L J M\right) \\
& \quad=4 \pi \sum_{L^{\prime \prime}} a_{L^{\prime}, L^{\prime \prime}} G_{L^{\prime \prime}}\left(q_{f}, q_{i}\right) a_{L^{\prime \prime}, L} \delta_{J^{\prime}, J} \delta_{M^{\prime}, M} .
\end{aligned}
$$

\section{LSJ representation operator}

Next we derive Eq. (A7). The spherical wave functions in momentum space with quantum numbers $J, L, S$ are for spin-0 spin-1/2 given by [27]

$$
\mathcal{Y}_{J L}^{M}(\hat{\mathbf{p}}, s)=\sum_{m, \mu} C_{m \mu M}^{L \frac{1}{2} J} Y_{m}^{L}(\hat{\mathbf{p}}) \chi_{\mu}^{\left(\frac{1}{2}\right)}(s),
$$

where $\chi$ is the baryon spin wave function. Using the definition for $\mathcal{Y}_{J L}^{M}$, Eq. (A9), we have

$$
\begin{aligned}
\sum_{s}\left(s^{\prime}|(\boldsymbol{\sigma} \cdot \hat{\mathbf{p}})| s\right) \mathcal{Y}_{J L}^{M}(\hat{\mathbf{p}}, s) \\
\quad=(-)^{m} \hat{\mathbf{p}}_{m}\left(s^{\prime}\left|\sigma_{-m}\right| s\right) C_{m_{l} \mu M}^{L \frac{1}{2} J} Y_{m_{l}}^{L}(\hat{\mathbf{p}}) \chi_{\mu}^{\left(\frac{1}{2}\right)}(s) \\
\quad=(-)^{m} \hat{\mathbf{p}}_{m}\left(s^{\prime}\left|\sigma_{-m}\right| s\right) C_{m_{l} s M}^{L \frac{1}{2} J} Y_{m_{l}}^{L}(\hat{\mathbf{p}}),
\end{aligned}
$$

where we used the convention of summation over repeated indices and quantization along the $z$ axis, which defines the spin variables $s, s^{\prime}$. Now, we use the expressions

$$
\begin{aligned}
& \hat{\mathbf{p}}_{m} Y_{m_{l}}^{L}(\hat{\mathbf{p}})=\sqrt{\frac{4 \pi}{3}} Y_{m}^{1}(\hat{\mathbf{p}}) Y_{m_{l}}^{L}(\hat{\mathbf{p}})
\end{aligned}
$$

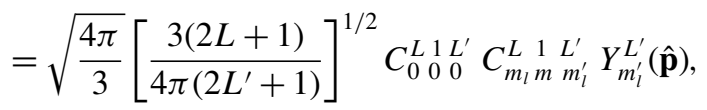

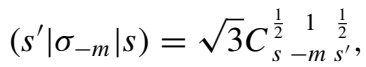

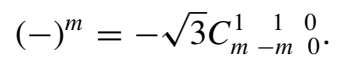

From the definition of the $9 j$ coefficient [28], these formulas give for Eq. (A10) the result

$$
\begin{aligned}
& \sum_{s}\left(s^{\prime}|(\boldsymbol{\sigma} \cdot \hat{\mathbf{p}})| s\right) \mathcal{Y}_{J L}^{M}(\hat{\mathbf{p}}, s)
\end{aligned}
$$

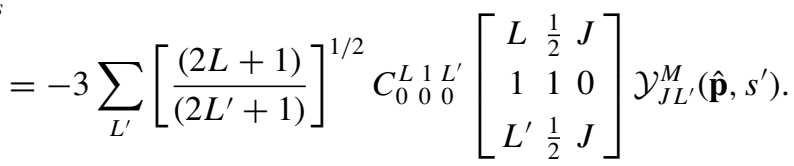

Evaluating Eq. (A12), one finds for the matrix $a$ in Eq. (A7),

$$
a=\left(\begin{array}{cc}
0 & -1 \\
-1 & 0
\end{array}\right)
$$

\section{APPENDIX B: RELATIVISTIC INVARIANT AMPLITUDES}

In this appendix the contributions from the various Feynman diagrams to the relativistic invariant amplitudes $A_{f i}(s, t, u)$ and $B_{f i}(s, t, u)$, defined in Eq. (2.5), are given. The results are valid for elastic as well as inelastic reactions. For details of the derivation we refer to [29].

\section{Momentum-space baryon-exchange diagrams}

(a). $J^{P}=1 / 2^{+}$baryon exchange.

(i) For pseudoscalar coupling

$$
\begin{aligned}
A_{p s} & =-\frac{g_{14} g_{23}}{u-M_{B}^{2}+i \epsilon}\left[-\frac{M_{f}+M_{i}}{2}+M_{B}\right], \\
B_{p s} & =-\frac{g_{14} g_{23}}{u-M_{B}^{2}+i \epsilon} .
\end{aligned}
$$


(ii) For pseudovector coupling

$$
\begin{aligned}
A_{p v}= & -\frac{f_{14} f_{23} / m_{\pi^{+}}^{2}}{u-M_{B}^{2}+i \epsilon}\left[u\left(\frac{M_{f}+M_{i}}{2}+M_{B}\right)\right. \\
& \left.-\frac{M_{f}+M_{i}}{2} M_{f} M_{i}-\frac{M_{f}^{2}+M_{i}^{2}}{2} M_{B}\right], \\
B_{p v}= & -\frac{f_{14} f_{23} / m_{\pi^{+}}^{2}}{u-M_{B}^{2}+i \epsilon} \\
& \times\left[u+\left(M_{f}+M_{i}\right) M_{B}+M_{f} M_{i}\right] .
\end{aligned}
$$

(b). $J^{P}=1 / 2^{+}$pole diagram. Using crossing symmetry [30], we can cross the results of the $u$-channel baryon exchange into the $s$ channel and obtain the invariant amplitudes $A_{f i}(s, t, u)$ and $B_{f i}(s, t, u)$ for the pole diagram. We have to replace $q \rightarrow-q^{\prime}$ and $q^{\prime} \rightarrow-q$, which means that we have to make the substitutions $u \leftrightarrow s, m_{f}^{2} \leftrightarrow m_{i}^{2}$ and add a minus sign to the amplitude $B$ because of Eq. (2.5). The $J^{P}=1 / 2^{+}$ pole amplitudes are given by the following:

(i) For pseudoscalar coupling

$$
\begin{aligned}
A_{p s} & =-\frac{g_{12} g_{34}}{s-M_{B}^{2}+i \epsilon}\left[-\frac{M_{f}+M_{i}}{2}+M_{B}\right], \\
B_{p s} & =\frac{g_{12} g_{34}}{s-M_{B}^{2}+i \epsilon} .
\end{aligned}
$$

(ii) For pseudovector coupling:

$$
\begin{gathered}
A_{p v}=-\frac{f_{12} f_{34} / m_{\pi^{+}}^{2}}{s-M_{B}^{2}+i \epsilon}\left[s\left(\frac{M_{f}+M_{i}}{2}+M_{B}\right)\right. \\
\left.-\frac{M_{f}+M_{i}}{2} M_{f} M_{i}-\frac{M_{f}^{2}+M_{i}^{2}}{2} M_{B}\right], \\
B_{p v}=\frac{f_{12} f_{34} / m_{\pi^{+}}^{2}}{s-M_{B}^{2}+i \epsilon}\left[s+\left(M_{f}+M_{i}\right) M_{B}+M_{f} M_{i}\right] . \\
\text { (c). } J^{P}=1 / 2^{-} \text {baryon exchange. }
\end{gathered}
$$

(i) For scalar coupling

$$
\begin{aligned}
& A_{s}=-\frac{g_{14}^{*(s)} g_{23}^{*(s)}}{u-M_{B}^{2}+i \epsilon}\left[-\frac{M_{f}+M_{i}}{2}-M_{B}\right], \\
& B_{s}=-\frac{g_{14}^{*(s)} g_{23}^{*(s)}}{u-M_{B}^{2}+i \epsilon} .
\end{aligned}
$$

(ii) For vector coupling

$$
\begin{aligned}
A_{v}= & -\frac{f_{14}^{*(v)} f_{23}^{*(v)} / m_{\pi^{+}}^{2}}{u-M_{B}^{2}+i \epsilon}\left[u\left(\frac{M_{f}+M_{i}}{2}-M_{B}\right)\right. \\
& \left.-\frac{M_{f}+M_{i}}{2} M_{f} M_{i}+\frac{M_{f}^{2}+M_{i}^{2}}{2} M_{B}\right], \\
B_{v}= & -\frac{f_{14}^{*(v)} f_{23}^{*(v)} / m_{\pi^{+}}^{2}}{u-M_{B}^{2}+i \epsilon} \\
& \times\left[u-\left(M_{f}+M_{i}\right) M_{B}+M_{f} M_{i}\right] .
\end{aligned}
$$

(d). $J^{P}=1 / 2^{-}$pole diagram. Applying crossing symmetry again we find, similar to the $J^{P}=1 / 2^{+}$baryon pole diagram, the invariant amplitudes for the $J^{P}=1 / 2^{-}$baryon pole diagram.

(i) For scalar coupling

$$
\begin{aligned}
A_{s} & =-\frac{g_{12}^{*(s)} g_{34}^{*(s)}}{s-M_{B}^{2}+i \epsilon}\left[-\frac{M_{f}+M_{i}}{2}-M_{B}\right], \\
B_{s} & =\frac{g_{12}^{*(s)} g_{34}^{*(s)}}{s-M_{B}^{2}+i \epsilon} .
\end{aligned}
$$

(ii) For vector coupling

$$
\begin{aligned}
A_{v}= & -\frac{f_{12}^{*(v)} f_{34}^{*(v)} / m_{\pi^{+}}^{2}}{s-M_{B}^{2}+i \epsilon}\left[s\left(\frac{M_{f}+M_{i}}{2}-M_{B}\right)\right. \\
& \left.-\frac{M_{f}+M_{i}}{2} M_{f} M_{i}+\frac{M_{f}^{2}+M_{i}^{2}}{2} M_{B}\right], \\
B_{v}= & \frac{f_{12}^{*(v)} f_{34}^{*(v)} / m_{\pi^{+}}^{2}}{s-M_{B}^{2}+i \epsilon} \\
& \times\left[s-\left(M_{f}+M_{i}\right) M_{B}+M_{f} M_{i}\right] .
\end{aligned}
$$

(e). $J^{P}=3 / 2^{+}$baryon exchange.

$$
\begin{aligned}
A_{Y^{*}}= & \frac{f_{14}^{*} f_{23}^{*} / m_{\pi^{+}}^{2}}{u-M_{Y^{*}}^{2}+i \epsilon} \\
& \times\left[\frac{t-m_{f}^{2}-m_{i}^{2}}{2}\left[\frac{M_{f}+M_{i}}{2}+M_{Y^{*}}\right]\right. \\
& +\frac{1}{6 M_{Y^{*}}^{2}}\left[M_{f}^{2}-m_{i}^{2}-u\right]\left[M_{i}^{2}-m_{f}^{2}-u\right] \\
& \times \frac{M_{f}+M_{i}}{2}+\frac{M_{Y^{*}}}{3}\left[u-\frac{M_{f}^{2}+M_{i}^{2}}{2}\right] \\
& +\frac{1}{3}\left[\frac{M_{f}+M_{i}}{2} u-\frac{\left(M_{f}^{2}+M_{i}^{2}\right)\left(M_{f}+M_{i}\right)}{4}\right. \\
& +\frac{\left.\left(m_{i}^{2}-m_{f}^{2}\right)\left(M_{f}-M_{i}\right)\right]+\frac{1}{4}}{6 M_{Y^{*}}} \\
& \times\left[\left(M_{f}^{2}-m_{i}^{2}-u\right)\left(\frac{M_{i}}{2}\left(M_{i}-M_{f}\right)-m_{f}^{2}\right)\right. \\
& \left.\left.-\left(M_{i}^{2}-m_{f}^{2}-u\right)\left(\frac{M_{f}}{2}\left(M_{i}-M_{f}\right)+m_{i}^{2}\right)\right]\right],
\end{aligned}
$$

$$
\begin{aligned}
B_{Y^{*}}= & \frac{f_{14}^{*} f_{23}^{*} / m_{\pi^{+}}^{2}}{u-M_{Y^{*}}^{2}+i \epsilon}\left[-\frac{t-m_{f}^{2}-m_{i}^{2}}{2}\right. \\
& -\frac{1}{6 M_{Y^{*}}^{2}}\left[u-M_{f}^{2}+m_{i}^{2}\right]\left[u-M_{i}^{2}+m_{f}^{2}\right] \\
& +\frac{M_{Y^{*}}}{3}\left(M_{f}+M_{i}\right)-\frac{m_{f}^{2}+m_{i}^{2}-\left(M_{f}+M_{i}\right)^{2}}{6}
\end{aligned}
$$




$$
\begin{aligned}
& +\frac{1}{6 M_{Y^{*}}}\left[M_{f}\left(M_{i}^{2}-m_{f}^{2}-u\right)\right. \\
& \left.\left.+M_{i}\left(M_{f}^{2}-m_{i}^{2}-u\right)\right]\right] .
\end{aligned}
$$

(f). $J^{P}=3 / 2^{+}$pole diagram. Applying crossing symmetry again we find, similar to the $J^{P}=1 / 2^{+}$baryon pole diagram, the following invariant amplitudes for the $J^{P}=3 / 2^{+}$ baryon pole diagram:

$$
\begin{aligned}
& A_{Y^{*}}= \frac{f_{12}^{*} f_{34}^{*} / m_{\pi^{+}}^{2}}{s-M_{Y^{*}}^{2}+i \epsilon} \\
& \times {\left[\frac{t-m_{f}^{2}-m_{i}^{2}}{2}\left[\frac{M_{f}+M_{i}}{2}+M_{Y^{*}}\right]\right.} \\
&+\frac{1}{6 M_{Y^{*}}^{2}}\left[M_{f}^{2}-m_{f}^{2}-s\right]\left[M_{i}^{2}-m_{i}^{2}-s\right] \\
& \times \frac{M_{f}+M_{i}}{2}+\frac{M_{Y^{*}}}{3}\left[s-\frac{M_{f}^{2}+M_{i}^{2}}{2}\right] \\
&+\frac{1}{3}\left[\frac{M_{f}+M_{i}}{2} s-\frac{\left(M_{f}^{2}+M_{i}^{2}\right)\left(M_{f}+M_{i}\right)}{4}\right. \\
&+\frac{\left.\left(m_{f}^{2}-m_{i}^{2}\right)\left(M_{f}-M_{i}\right)\right]+\frac{1}{4}}{6 M_{Y^{*}}} \\
& \times {\left[\left(M_{f}^{2}-m_{f}^{2}-s\right)\left(\frac{M_{i}}{2}\left(M_{i}-M_{f}\right)-m_{i}^{2}\right)\right.} \\
&+ \frac{1}{6 M_{Y^{*}}}\left[M_{i}\left(M_{f}^{2}-m_{f}^{2}-s\right)\right] \\
& B_{Y^{*}}=- \frac{M_{Y^{*}}^{*} f_{34}^{*} / m_{\pi^{+}}^{2}}{s-M_{Y^{*}}^{2}+i \epsilon}\left[-\frac{\left.\left.t-m_{f}^{2}-s\right)\left(\frac{M_{f}}{2}\left(M_{i}-M_{f}\right)+m_{i}^{2}\right)\right]}{2}\right. \\
&- \frac{1}{6 M_{Y}^{2}}\left[s-M_{f}^{2}+m_{f}^{2}\right]\left[s-M_{i}^{2}+m_{i}^{2}\right] \\
& \hline
\end{aligned}
$$

\section{Momentum-space meson-exchange diagrams}

(a). $J^{P}=0^{++}$scalar-meson exchange.

$$
\begin{aligned}
A_{S} & =\frac{g_{P P S} g_{S}}{t-m_{S}^{2}+i \epsilon}, \\
B_{S} & =0 .
\end{aligned}
$$

(b). $J^{P}=1^{--}$vector-meson exchange.

$$
\begin{aligned}
& A_{V}=\frac{g_{P P V}}{t-m_{V}^{2}+i \epsilon}\left[g_{V} \frac{m_{f}^{2}-m_{i}^{2}}{m_{V}^{2}}\right. \\
&\left.\times\left(M_{i}-M_{f}\right)+\frac{s-u}{2 \mathcal{M}} f_{V}\right], \\
& B_{V}=-2 \frac{g_{P P V}}{t-m_{V}^{2}+i \epsilon}\left[f_{V} \frac{M_{f}+M_{i}}{2 \mathcal{M}}+g_{V}\right] .
\end{aligned}
$$

(c). $J^{P}=2^{++}$tensor-meson exchange.

$$
\begin{aligned}
A_{T}= & \frac{g_{P P T} / m_{\pi^{+}}}{\Delta^{2}-m_{T}^{2}+i \epsilon}\left[\left(\frac{s-u}{2}\right)^{2} F_{2}\right. \\
& -\frac{1}{2 m_{T}^{2}}\left(m_{f}^{2}-m_{i}^{2}\right)(s-u)\left[\left(M_{i}-M_{f}\right) F_{1}\right. \\
& \left.+\left(M_{i}^{2}-M_{f}^{2}\right) F_{2}\right]+\frac{1}{2 m_{T}^{4}}\left(m_{f}^{2}-m_{i}^{2}\right)^{2} \\
& \times\left(M_{i}^{2}-M_{f}^{2}\right)\left[\left(M_{i}-M_{f}\right) F_{1}+\frac{M_{i}^{2}-M_{f}^{2}}{2} F_{2}\right] \\
& -\frac{4}{3}\left[-Q^{2}+\frac{1}{4 m_{T}^{2}}\left(m_{f}^{2}-m_{i}^{2}\right)^{2}\right] \\
& \times\left[-\frac{1}{2}\left(\left(M_{f}+M_{i}\right) F_{1}+\frac{1}{2}\left(p^{\prime}+p\right)^{2} F_{2}\right)\right. \\
& +\frac{M_{i}^{2}-M_{f}^{2}}{2 m_{T}^{2}}\left(\left(M_{i}-M_{f}\right) F_{1}\right. \\
& \left.\left.\left.+\frac{1}{2}\left(M_{i}^{2}-M_{f}^{2}\right) F_{2}\right)\right]\right], \\
B_{T}= & \frac{g_{P P T} / m_{\pi^{+}}}{\Delta^{2}-m_{T}^{2}+i \epsilon}\left[(s-u) F_{1}\right.
\end{aligned}
$$

\section{Momentum-space Pomeron exchange}

$$
\begin{aligned}
A_{P} & =\frac{g_{P P P} g_{P}}{\mathcal{M}}, \\
B_{P} & =0 .
\end{aligned}
$$

\section{APPENDIX C: $X, Y, Z$ COEFFICIENTS}

Here we list the explicit expressions for the expansion coefficients $X^{(\alpha)}, Y^{(\alpha)}, Z^{(\alpha)}$, and $U^{(\alpha)}$ of the partial wave 
potentials, Eq. (4.23), for each type of exchange in the $s, u$, and $t$ channels. We have introduced the notation $N_{f i}^{ \pm}=$ $\sqrt{\left(E_{i} \pm M_{i}\right)\left(E_{f} \pm M_{f}\right)}$.

\section{Baryon-exchange}

(a). $J^{P}=1 / 2^{+}$baryon exchange.

(i) For pseudoscalar coupling

$$
\begin{aligned}
X_{B}^{(C)} & =g_{14} g_{23} N_{f i}^{+}\left[M_{B}+\frac{W_{f}+W_{i}}{2}-M_{f}-M_{i}\right], \\
Y_{B}^{(C)} & =g_{14} g_{23} N_{f i}^{-}\left[-M_{B}+\frac{W_{f}+W_{i}}{2}+M_{f}+M_{i}\right], \\
X_{B}^{(\mathrm{SO})} & =-g_{14} g_{23} N_{f i}^{-}\left[-M_{B}+\frac{W_{f}+W_{i}}{2}+M_{f}+M_{i}\right] .
\end{aligned}
$$

(ii) For pseudovector coupling

$$
\begin{aligned}
& X_{B}^{(C)}=-\frac{f_{14} f_{23}}{m_{\pi^{+}}^{2}} N_{f i}^{+}\left[\left(-\frac{M_{f}+M_{i}}{2}-M_{B}\right)\right. \\
& \times\left(\left(\frac{E_{f}+E_{i}-\omega_{f}-\omega_{i}}{2}\right)^{2}-p_{f}^{2}-p_{i}^{2}\right) \\
& +\frac{M_{f}+M_{i}}{2} M_{f} M_{i}+\frac{M_{f}^{2}+M_{i}^{2}}{2} M_{B} \\
& -\frac{W_{f}+W_{i}-M_{f}-M_{i}}{2} \\
& \times\left(\left(\frac{E_{f}+E_{i}-\omega_{f}-\omega_{i}}{2}\right)^{2}-p_{f}^{2}-p_{i}^{2}\right. \\
& \left.\left.+\left(M_{f}+M_{i}\right) M_{B}+M_{f} M_{i}\right)\right], \\
& Y_{B}^{(C)}=-\frac{f_{14} f_{23}}{m_{\pi^{+}}^{2}}\left[N _ { f i } ^ { + } \left[-\left(-\frac{M_{f}+M_{i}}{2}-M_{B}\right)\right.\right. \\
& \left.+\frac{W_{f}+W_{i}-M_{f}-M_{i}}{2}\right] 2 p_{f} p_{i}+N_{f i}^{-} \\
& \times\left[-\left(-\frac{M_{f}+M_{i}}{2}-M_{B}\right)\right. \\
& \times\left(\left(\frac{E_{f}+E_{i}-\omega_{f}-\omega_{i}}{2}\right)^{2}-p_{f}^{2}-p_{i}^{2}\right) \\
& -\frac{M_{f}+M_{i}}{2} M_{f} M_{i}-\frac{M_{f}^{2}+M_{i}^{2}}{2} M_{B} \\
& -\frac{W_{f}+W_{i}+M_{f}+M_{i}}{2}\left(\left(\frac{E_{f}+E_{i}-\omega_{f}-\omega_{i}}{2}\right)^{2}\right. \\
& \left.\left.\left.-p_{f}^{2}-p_{i}^{2}+\left(M_{f}+M_{i}\right) M_{B}+M_{f} M_{i}\right)\right]\right] \text {, } \\
& Z_{B}^{(C)}=-\frac{f_{14} f_{23}}{m_{\pi^{+}}^{2}} N_{f i}^{-}\left[-\frac{M_{f}+M_{i}}{2}\right. \\
& \left.-M_{B}+\frac{W_{f}+W_{i}+M_{f}+M_{i}}{2}\right] 2 p_{f} p_{i},
\end{aligned}
$$

$$
\begin{aligned}
X_{B}^{(\mathrm{SO})}= & \frac{f_{14} f_{23}}{m_{\pi^{+}}^{2}} N_{f i}^{-}\left[-\left(-\frac{M_{f}+M_{i}}{2}-M_{B}\right)\right. \\
& \times\left(\left(\frac{E_{f}+E_{i}-\omega_{f}-\omega_{i}}{2}\right)^{2}-p_{f}^{2}-p_{i}^{2}\right) \\
& -\frac{M_{f}+M_{i}}{2} M_{f} M_{i}-\frac{M_{f}^{2}+M_{i}^{2}}{2} M_{B} \\
& -\frac{W_{f}+W_{i}+M_{f}+M_{i}}{2}\left(\left(\frac{E_{f}+E_{i}-\omega_{f}-\omega_{i}}{2}\right)^{2}\right. \\
& \left.\left.-p_{f}^{2}-p_{i}^{2}+\left(M_{f}+M_{i}\right) M_{B}+M_{f} M_{i}\right)\right], \\
Y_{B}^{(\mathrm{SO})}= & \frac{f_{14} f_{23}}{m_{\pi^{+}}^{2}} N_{f i}^{-}\left[-\frac{M_{f}+M_{i}}{2}-M_{B}\right. \\
& \left.+\frac{W_{f}+W_{i}+M_{f}+M_{i}}{2}\right] 2 p_{f} p_{i} .
\end{aligned}
$$

(b). $J^{P}=1 / 2^{+}$pole term.

(i) For pseudoscalar coupling

$$
\begin{aligned}
X_{B}^{(C)} & =-g_{12} g_{34} N_{f i}^{+}\left[M_{B}-\frac{W_{f}+W_{i}}{2}\right], \\
Y_{B}^{(C)} & =g_{12} g_{34} N_{f i}^{-}\left[M_{B}+\frac{W_{f}+W_{i}}{2}\right], \\
X_{B}^{(\mathrm{SO})} & =-g_{12} g_{34} N_{f i}^{-}\left[M_{B}+\frac{W_{f}+W_{i}}{2}\right] .
\end{aligned}
$$

(ii) For pseudovector coupling

$$
\begin{aligned}
X_{B}^{(C)}= & \frac{f_{12} f_{34}}{m_{\pi^{+}}^{2}} N_{f i}^{+}\left[\left(-\frac{M_{f}+M_{i}}{2}-M_{B}\right) s\right. \\
& +\frac{M_{f}+M_{i}}{2} M_{f} M_{i}+\frac{W_{f}+W_{i}-M_{f}-M_{i}}{2} \\
& \times\left[s+\left(M_{f}+M_{i}\right) M_{B}+M_{f} M_{i}\right] \\
& \left.+\frac{M_{f}^{2}+M_{i}^{2}}{2} M_{B}\right], \\
Y_{B}^{(C)}= & \frac{f_{12} f_{34}}{m_{\pi^{+}}^{2}} N_{f i}^{-}\left[-\left(-\frac{M_{f}+M_{i}}{2}-M_{B}\right) s\right. \\
& -\frac{M_{f}+M_{i}}{2} M_{f} M_{i}+\frac{W_{f}+W_{i}-M_{f}-M_{i}}{2} \\
& \times\left[s+\left(M_{f}+M_{i}\right) M_{B}+M_{f} M_{i}\right] \\
& \left.-\frac{M_{f}^{2}+M_{i}^{2}}{2} M_{B}\right], \\
X_{B}^{(\mathrm{SO})}= & -\frac{f_{12} f_{34}}{m_{\pi^{+}}^{2}} N_{f i}^{-}\left[-\left(-\frac{M_{f}+M_{i}}{2}-M_{B}\right) s\right. \\
& -\frac{M_{f}+M_{i}}{2} M_{f} M_{i}+\frac{W_{f}+W_{i}-M_{f}-M_{i}}{2} \\
& \times\left[s+\left(M_{f}+M_{i}\right) M_{B}+M_{f} M_{i}\right] \\
& \left.-\frac{M_{f}^{2}+M_{i}^{2}}{2} M_{B}\right] .
\end{aligned}
$$


(c). $J^{P}=1 / 2^{-}$baryon exchange.

(i) For scalar coupling

$$
\begin{aligned}
X_{B}^{(C)} & =g_{14}^{*(s)} g_{23}^{*(s)} N_{f i}^{+}\left[-M_{B}+\frac{W_{f}+W_{i}}{2}-M_{f}-M_{i}\right], \\
Y_{B}^{(C)} & =g_{14}^{*(s)} g_{23}^{*(s)} N_{f i}^{-}\left[M_{B}+\frac{W_{f}+W_{i}}{2}+M_{f}+M_{i}\right], \\
X_{B}^{(\mathrm{SO})} & =-g_{14}^{*(s)} g_{23}^{*(s)} N_{f i}^{-}\left[M_{B}+\frac{W_{f}+W_{i}}{2}+M_{f}+M_{i}\right] .
\end{aligned}
$$

(ii) For vector coupling

$$
\begin{aligned}
& X_{B}^{(C)}=-\frac{f_{14}^{*(v)} f_{23}^{*(v)}}{m_{\pi^{+}}^{2}} N_{f i}^{+}\left[\left(-\frac{M_{f}+M_{i}}{2}+M_{B}\right)\right. \\
& \times\left(\left(\frac{E_{f}+E_{i}-\omega_{f}-\omega_{i}}{2}\right)^{2}-p_{f}^{2}-p_{i}^{2}\right) \\
& +\frac{M_{f}+M_{i}}{2} M_{f} M_{i}-\frac{M_{f}^{2}+M_{i}^{2}}{2} M_{B} \\
& -\frac{W_{f}+W_{i}-M_{f}-M_{i}}{2}\left(\left(\frac{E_{f}+E_{i}-\omega_{f}-\omega_{i}}{2}\right)^{2}\right. \\
& \left.\left.-p_{f}^{2}-p_{i}^{2}-\left(M_{f}+M_{i}\right) M_{B}+M_{f} M_{i}\right)\right] \text {, } \\
& Y_{B}^{(C)}=-\frac{f_{14}^{*(v)} f_{23}^{*(v)}}{m_{\pi^{+}}^{2}}\left[N _ { f i } ^ { + } \left[-\left(-\frac{M_{f}+M_{i}}{2}+M_{B}\right)\right.\right. \\
& \left.+\frac{W_{f}+W_{i}-M_{f}-M_{i}}{2}\right] 2 p_{f} p_{i}+N_{f i}^{-} \\
& \times\left[-\left(-\frac{M_{f}+M_{i}}{2}+M_{B}\right)\right. \\
& \times\left(\left(\frac{E_{f}+E_{i}-\omega_{f}-\omega_{i}}{2}\right)^{2}-p_{f}^{2}-p_{i}^{2}\right) \\
& -\frac{M_{f}+M_{i}}{2} M_{f} M_{i}+\frac{M_{f}^{2}+M_{i}^{2}}{2} M_{B} \\
& -\frac{W_{f}+W_{i}+M_{f}+M_{i}}{2}\left(\left(\frac{E_{f}+E_{i}-\omega_{f}-\omega_{i}}{2}\right)^{2}\right. \\
& \left.\left.\left.-p_{f}^{2}-p_{i}^{2}-\left(M_{f}+M_{i}\right) M_{B}+M_{f} M_{i}\right)\right]\right], \\
& Z_{B}^{(C)}=-\frac{f_{14}^{*(v)} f_{23}^{*(v)}}{m_{\pi^{+}}^{2}} N_{f i}^{-}\left[-\frac{M_{f}+M_{i}}{2}+M_{B}\right. \\
& \left.+\frac{W_{f}+W_{i}+M_{f}+M_{i}}{2}\right] 2 p_{f} p_{i}, \\
& X_{B}^{(\mathrm{SO})}=\frac{f_{14}^{*(v)} f_{23}^{*(v)}}{m_{\pi^{+}}^{2}} N_{f i}^{-}\left[-\left(-\frac{M_{f}+M_{i}}{2}+M_{B}\right)\right. \\
& \times\left(\left(\frac{E_{f}+E_{i}-\omega_{f}-\omega_{i}}{2}\right)^{2}-p_{f}^{2}-p_{i}^{2}\right)
\end{aligned}
$$

$$
\begin{aligned}
& -\frac{M_{f}+M_{i}}{2} M_{f} M_{i}+\frac{M_{f}^{2}+M_{i}^{2}}{2} M_{B} \\
& -\frac{W_{f}+W_{i}+M_{f}+M_{i}}{2}\left(\left(\frac{E_{f}+E_{i}-\omega_{f}-\omega_{i}}{2}\right)^{2}\right. \\
& \left.\left.-p_{f}^{2}-p_{i}^{2}-\left(M_{f}+M_{i}\right) M_{B}+M_{f} M_{i}\right)\right], \\
Y_{B}^{(\mathrm{SO})}= & \frac{f_{14}^{*(v)} f_{23}^{*(v)}}{m_{\pi^{+}}^{2}} N_{f i}^{-}\left[-\frac{M_{f}+M_{i}}{2}+M_{B}\right. \\
& \left.+\frac{W_{f}+W_{i}+M_{f}+M_{i}}{2}\right] 2 p_{f} p_{i} .
\end{aligned}
$$

(d). $J^{P}=1 / 2^{-}$pole term.

(i) For scalar coupling

$$
\begin{aligned}
X_{B}^{(C)} & =g_{12}^{*(v)} g_{34}^{*(v)} N_{f i}^{+}\left[M_{B}+\frac{W_{f}+W_{i}}{2}\right], \\
Y_{B}^{(C)} & =g_{12}^{*(v)} g_{34}^{*(v)} N_{f i}^{-}\left[-M_{B}+\frac{W_{f}+W_{i}}{2}\right], \\
X_{B}^{(\mathrm{SO})} & =-g_{12}^{*(v)} g_{34}^{*(v)} N_{f i}^{-}\left[-M_{B}+\frac{W_{f}+W_{i}}{2}\right] .
\end{aligned}
$$

(ii) For vector coupling

$$
\begin{aligned}
X_{B}^{(C)}= & \frac{f_{12}^{*(v)} f_{34}^{*(v)}}{m_{\pi^{+}}^{2}} N_{f i}^{+}\left[\left(-\frac{M_{f}+M_{i}}{2}+M_{B}\right) s\right. \\
& +\frac{M_{f}+M_{i}}{2} M_{f} M_{i}+\frac{W_{f}+W_{i}-M_{f}-M_{i}}{2} \\
& \times\left[s-\left(M_{f}+M_{i}\right) M_{B}+M_{f} M_{i}\right] \\
& \left.-\frac{M_{f}^{2}+M_{i}^{2}}{2} M_{B}\right], \\
Y_{B}^{(C)}= & \frac{f_{12}^{*(v)} f_{34}^{*(v)}}{m_{\pi^{+}}^{2}} N_{f i}^{-}\left[-\left(-\frac{M_{f}+M_{i}}{2}+M_{B}\right) s\right. \\
& -\frac{M_{f}+M_{i}}{2} M_{f} M_{i}+\frac{W_{f}+W_{i}-M_{f}-M_{i}}{2} \\
& \times\left[s-\left(M_{f}+M_{i}\right) M_{B}+M_{f} M_{i}\right] \\
& \left.+\frac{M_{f}^{2}+M_{i}^{2}}{2} M_{B}\right], \\
X_{B}^{(\mathrm{SO})}= & -\frac{f_{12}^{*(v)} f_{34}^{*(v)}}{m_{\pi^{+}}^{2}} N_{f i}^{-}\left[-\left(-\frac{M_{f}+M_{i}}{2}+M_{B}\right) s\right. \\
& -\frac{M_{f}+M_{i}}{2} M_{f} M_{i}+\frac{W_{f}+W_{i}-M_{f}-M_{i}}{2} \\
& \times\left[s-\left(M_{f}+M_{i}\right) M_{B}+M_{f} M_{i}\right] \\
& \left.+\frac{M_{f}^{2}+M_{i}^{2}}{2} M_{B}\right] .
\end{aligned}
$$

(e). $J^{P}=3 / 2^{+}$baryon exchange.

$X_{Y^{*}}^{(C)}=-\frac{f_{14}^{*} f_{23}^{*}}{m_{\pi^{+}}^{2}} N_{f i}^{+}\left[A_{0}+\frac{B_{0}}{2}\left(W_{f}+W_{i}-M_{i}-M_{f}\right)\right]$, 


$$
\begin{aligned}
Y_{Y^{*}}^{(C)}= & -\frac{f_{14}^{*} f_{23}^{*}}{m_{\pi^{+}}^{2}}\left[N_{f i}^{+}\left[A_{1}+\frac{B_{1}}{2}\left(W_{f}+W_{i}-M_{f}-M_{i}\right)\right]\right. \\
& \left.+N_{f i}^{-}\left[-A_{0}+\frac{B_{0}}{2}\left(W_{f}+W_{i}+M_{f}+M_{i}\right)\right]\right], \\
Z_{Y^{*}}^{(C)}= & -\frac{f_{14}^{*} f_{23}^{*}}{m_{\pi^{+}}^{2}}\left[N_{f i}^{+}\left[A_{2}+\frac{B_{2}}{2}\left(W_{f}+W_{i}-M_{f}-M_{i}\right)\right]\right. \\
& \left.+N_{f i}^{-}\left[-A_{1}+\frac{B_{1}}{2}\left(W_{f}+W_{i}+M_{f}+M_{i}\right)\right]\right], \\
U_{Y^{*}}^{(C)}= & \frac{f_{14}^{*} f_{23}^{*}}{m_{\pi^{+}}^{2}} N_{f i}^{-}\left[-A_{2}+\frac{B_{2}}{2}\left(W_{f}+W_{i}+M_{f}+M_{i}\right)\right], \\
X_{Y^{*}}^{(\mathrm{SO})}= & \frac{f_{14}^{*} f_{23}^{*}}{m_{\pi^{+}}^{2}} N_{f i}^{-}\left[-A_{0}+\frac{B_{0}}{2}\left(W_{f}+W_{i}+M_{i}+M_{f}\right)\right], \\
Y_{Y^{*}}^{(\mathrm{SO})}= & \frac{f_{14}^{*} f_{23}^{*}}{m_{\pi^{+}}^{2}} N_{f i}^{-}\left[-A_{1}+\frac{B_{1}}{2}\left(W_{f}+W_{i}+M_{i}+M_{f}\right)\right], \\
Z_{Y^{*}}^{(\mathrm{SO})}= & \frac{f_{14}^{*} f_{23}^{*}}{m_{\pi^{+}}^{2}} N_{f i}^{-}\left[-A_{2}+\frac{B_{0}}{2}\left(W_{f}+W_{i}-M_{f}-M_{i}\right)\right],
\end{aligned}
$$

where $A_{0}, A_{1}, A_{2}, B_{0}, B_{1}$, and $B_{2}$ depend on the mass and momentum of the particles as follows:

$$
\begin{aligned}
& A_{0}=\frac{1}{12 M_{Y^{*}}^{2}}\left(M_{f}+M_{i}\right)\left(-2 p_{f} p_{i} z_{u}+M_{Y^{*}}^{2}\right)^{2} \\
& +\left(-\frac{1}{6 M_{Y^{*}}^{2}}\left(M_{f}^{2}+M_{i}^{2}-m_{f}^{2}-m_{i}^{2}\right) \frac{M_{f}+M_{i}}{2}\right. \\
& +\frac{M_{Y^{*}}}{3}+\frac{M_{f}+M_{i}}{6}-\frac{1}{6 M_{Y^{*}}}\left(\frac{M_{i}}{2}\left(M_{i}-M_{f}\right)\right. \\
& \left.\left.-m_{f}^{2}+\frac{M_{f}}{2}\left(M_{f}-M_{i}\right)-m_{i}^{2}\right)\right)\left(-2 p_{f} p_{i} z_{u}+M_{Y^{*}}^{2}\right) \\
& +\frac{1}{2}\left(\frac{M_{f}+M_{i}}{2}+M_{Y^{*}}\right)\left(\frac{\left(E_{f}-E_{i}\right)^{2}+\left(\omega_{f}-\omega_{i}\right)^{2}}{2}\right. \\
& \left.-p_{f}^{2}-p_{i}^{2}\right)-\frac{m_{f}^{2}+m_{i}^{2}}{2}\left(\frac{M_{f}+M_{i}}{2}+M_{Y^{*}}\right) \\
& +\frac{1}{12 M_{Y^{*}}^{2}}\left(M_{f}+M_{i}\right)\left(M_{f}^{2}-m_{i}^{2}\right)\left(M_{i}^{2}-m_{f}^{2}\right) \\
& -\frac{M_{Y^{*}}}{6}\left(M_{f}^{2}+M_{i}^{2}\right)+\frac{1}{12}\left(\left(m_{i}^{2}-m_{f}^{2}\right)\left(M_{f}-M_{i}\right)\right. \\
& \left.-\left(M_{f}^{2}+M_{i}^{2}\right)\left(M_{f}+M_{i}\right)\right)+\frac{1}{6 M_{Y^{*}}}\left(\left(M_{f}^{2}-m_{i}^{2}\right)\right. \\
& \times\left(\frac{M_{i}}{2}\left(M_{i}-M_{f}\right)-m_{f}^{2}\right)+\left(M_{i}^{2}-m_{f}^{2}\right) \\
& \left.\times\left(\frac{M_{f}}{2}\left(M_{f}-M_{i}\right)-m_{i}^{2}\right)\right),
\end{aligned}
$$

$$
\begin{aligned}
A_{1}= & {\left[-\frac{1}{12 M_{Y^{*}}^{2}}\left(M_{f}+M_{i}\right) 2\left(-2 p_{f} p_{i} z_{u}+M_{Y^{*}}^{2}\right)\right.} \\
& +\frac{1}{6 M_{Y^{*}}^{2}}\left(M_{f}^{2}+M_{i}^{2}-m_{f}^{2}-m_{i}^{2}\right) \frac{M_{f}+M_{i}}{2} \\
& -\frac{M_{Y^{*}}}{3}-\frac{M_{f}+M_{i}}{6}+\frac{1}{6 M_{Y^{*}}}\left(\frac{M_{i}}{2}\left(M_{i}-M_{f}\right)\right. \\
& \left.-m_{f}^{2}+\frac{M_{f}}{2}\left(M_{f}-M_{i}\right)-m_{i}^{2}\right) \\
& \left.+\frac{1}{2}\left(\frac{M_{f}+M_{i}}{2}+M_{Y^{*}}\right)\right] 2 p_{f} p_{i},
\end{aligned}
$$$$
A_{2}=\frac{1}{12 M_{Y^{*}}^{2}}\left(M_{f}+M_{i}\right)\left(2 p_{f} p_{i}\right)^{2} \text {, }
$$$$
B_{0}=\frac{m_{f}^{2}+m_{i}^{2}}{2}-\frac{1}{6 M_{Y^{*}}^{2}}\left(M_{f}^{2}-m_{i}^{2}\right)\left(M_{i}^{2}-m_{f}^{2}\right)
$$$$
+\frac{M_{Y^{*}}}{3}\left(M_{f}+M_{i}\right)-\frac{m_{f}^{2}+m_{i}^{2}-\left(M_{f}+M_{i}\right)^{2}}{6}
$$$$
+\frac{1}{6 M_{Y^{*}}}\left(M_{f}\left(M_{i}^{2}-m_{f}^{2}\right)+M_{i}\left(M_{f}^{2}-m_{i}^{2}\right)\right)
$$$$
-\frac{1}{2}\left(\frac{\left(E_{f}-E_{i}\right)^{2}+\left(\omega_{f}-\omega_{i}\right)^{2}}{2}-p_{f}^{2}-p_{i}^{2}\right)
$$$$
-\frac{1}{6 M_{Y^{*}}^{2}}\left(-2 p_{f} p_{i} z_{u}+M_{Y^{*}}^{2}\right)^{2}
$$$$
+\left(\frac{1}{6 M_{Y^{*}}^{2}}\left(M_{f}^{2}+M_{i}^{2}-m_{f}^{2}-m_{i}^{2}\right)\right.
$$$$
\left.-\frac{1}{6 M_{Y^{*}}}\left(M_{f}+M_{i}\right)\right)\left(-2 p_{f} p_{i} z_{u}+M_{Y^{*}}^{2}\right),
$$$$
B_{1}=\left[\frac{1}{3 M_{Y^{*}}^{2}}\left(-2 p_{f} p_{i} z_{u}+M_{Y^{*}}^{2}\right)\right.
$$$$
-\frac{1}{6 M_{Y^{*}}}\left(\frac{1}{M_{Y^{*}}}\left(M_{f}^{2}+M_{i}^{2}-m_{f}^{2}-m_{i}^{2}\right)\right.
$$$$
\left.\left.-\left(M_{f}+M_{i}\right)\right)-\frac{1}{2}\right] 2 p_{f} p_{i},
$$$$
B_{2}=-\frac{1}{6 M_{Y^{*}}^{2}}\left(2 p_{f} p_{i}\right)^{2} .
$$

(f). $J^{P}=3 / 2^{+}$pole term.

$$
\begin{aligned}
X_{Y^{*}}^{(C)}= & \frac{f_{12}^{*} f_{34}^{*}}{m_{\pi^{+}}^{2}} N_{f i}^{+}\left[A_{0}+\frac{B_{0}}{2}\left(W_{f}+W_{i}-M_{i}-M_{f}\right)\right], \\
Y_{Y^{*}}^{(C)}= & \frac{f_{12}^{*} f_{34}^{*}}{m_{\pi^{+}}^{2}}\left[N_{f i}^{+}\left[A_{1}+\frac{B_{1}}{2}\left(W_{f}+W_{i}-M_{f}-M_{i}\right)\right]\right. \\
& \left.+N_{f i}^{-}\left[-A_{0}+\frac{B_{0}}{2}\left(W_{f}+W_{i}+M_{f}+M_{i}\right)\right]\right], \\
Z_{Y^{*}}^{(C)}= & \frac{f_{12}^{*} f_{34}^{*}}{m_{\pi^{+}}^{2}} N_{f i}^{-}\left[-A_{1}+\frac{B_{1}}{2}\right. \\
& \left.\times\left(W_{f}+W_{i}+M_{f}+M_{i}\right)\right],
\end{aligned}
$$


$X_{Y^{*}}^{(\mathrm{SO})}=-\frac{f_{12}^{*} f_{34}^{*}}{m_{\pi^{+}}^{2}} N_{f i}^{-}\left[-A_{0}+\frac{B_{0}}{2}\left(W_{f}+W_{i}+M_{i}+M_{f}\right)\right]$,
$Y_{Y^{*}}^{(\mathrm{SO})}=-\frac{f_{12}^{*} f_{34}^{*}}{m_{\pi^{+}}^{2}} N_{f i}^{-}\left[-A_{1}+\frac{B_{1}}{2}\left(W_{f}+W_{i}+M_{i}+M_{f}\right)\right]$.

where $A_{0}, A_{1}, B_{0}$, and $B_{1}$ depend on the mass and momentum of the particles as follows:

$$
\begin{aligned}
A_{0}= & \frac{1}{2}\left(\frac{\left(E_{f}-E_{i}\right)^{2}+\left(\omega_{f}-\omega_{i}\right)^{2}}{2}-p_{f}^{2}-p_{i}^{2}-m_{f}^{2}-m_{i}^{2}\right) \\
& \times\left(\frac{M_{f}+M_{i}}{2}+M_{Y^{*}}\right)+\frac{1}{6 M_{Y^{*}}^{2}}\left(M_{f}^{2}-m_{f}^{2}-s\right) \\
& \times\left(M_{i}^{2}-m_{i}^{2}-s\right) \frac{M_{f}+M_{i}}{2} \\
& +\frac{1}{3 M_{Y^{*}}}\left(s-\frac{1}{2}\left(M_{f}^{2}+M_{i}^{2}\right)\right)+\frac{1}{3}\left(\frac{M_{f}+M_{i}}{2} s\right. \\
& \left.-\frac{\left(M_{f}^{2}+M_{i}^{2}\right)\left(M_{f}+M_{i}\right)}{4}+\frac{m_{f}^{2}-m_{i}^{2}}{4}\left(M_{f}-M_{i}\right)\right) \\
& +\frac{1}{6 M_{Y^{*}}}\left(\left(M_{f}^{2}-m_{f}^{2}-s\right)\left(\frac{M_{i}}{2}\left(M_{i}-M_{f}\right)-m_{i}^{2}\right)\right. \\
& \left.-\left(M_{i}^{2}-m_{i}^{2}-s\right)\left(\frac{M_{f}}{2}\left(M_{i}-M_{f}\right)+m_{f}^{2}\right)\right), \\
A_{1}= & \frac{1}{2}\left[\frac{M_{f}+M_{i}}{2}+M_{Y^{*}}\right] 2 p_{f} p_{i},
\end{aligned}
$$

$$
\begin{aligned}
B_{0}= & \frac{1}{2}\left(\frac{\left(E_{f}-E_{i}\right)^{2}+\left(\omega_{f}-\omega_{i}\right)^{2}}{2}-p_{f}^{2}-p_{i}^{2}-m_{f}^{2}-m_{i}^{2}\right) \\
& -\frac{1}{6 M_{Y^{*}}^{2}}\left(s-M_{f}^{2}+m_{f}^{2}\right)\left(s-M_{i}^{2}+m_{i}^{2}\right) \\
& -\frac{M_{Y^{*}}}{3}\left(M_{f}+M_{i}\right)+\frac{m_{f}^{2}+m_{i}^{2}-\left(M_{f}+M_{i}\right)^{2}}{6} \\
& -\frac{1}{6 M_{Y^{*}}}\left(M_{f}\left(M_{i}^{2}-m_{i}^{2}-s\right)+M_{i}\left(M_{f}^{2}-m_{f}^{2}-s\right)\right),
\end{aligned}
$$

$B_{1}=p_{f} p_{i}$.

\section{Meson exchange}

(a). $J^{P}=0^{++}$scalar-meson exchange.

$$
\begin{gathered}
X_{S}^{(C)}=-g_{P P S} g_{S} N_{f i}^{+}, \\
Y_{S}^{(C)}=g_{P P S} g_{S} N_{f i}^{-}, \\
X_{S}^{(\mathrm{SO})}=-g_{P P S} g_{S} N_{f i}^{-} .
\end{gathered}
$$

(b). $J^{P}=1^{-}$vector-meson exchange.

$$
\begin{aligned}
X_{V}^{(C)}= & -g_{P P V} g_{V} N_{f i}^{+}\left[\frac{\left(m_{f}^{2}-m_{i}^{2}\right)\left(M_{i}-M_{f}\right)}{m_{V}^{2}}\right. \\
& \left.-\left(W_{f}+W_{i}-M_{i}-M_{f}\right)\right]-g_{P P V} f_{V} N_{f i}^{+}
\end{aligned}
$$

$$
\begin{aligned}
\times & {\left[-\frac{M_{f}+M_{i}}{2 \mathcal{M}}\left(W_{f}+W_{i}-M_{i}-M_{f}\right)\right.} \\
& \left.+\frac{\left.\left(\omega_{f}+\omega_{i}\right)\left(E_{f}+E_{i}\right)+p_{f}^{2}+p_{i}^{2}\right]}{2 \mathcal{M}}\right], \\
Y_{V}^{(C)}= & -g_{P P V} g_{V} N_{f i}^{-}\left[-\frac{\left(m_{f}^{2}-m_{i}^{2}\right)\left(M_{i}-M_{f}\right)}{m_{V}^{2}}\right. \\
& \left.-\left(W_{f}+W_{i}+M_{i}+M_{f}\right)\right]-g_{P P V} f_{V}\left[N_{f i}^{+} \frac{p_{f} p_{i}}{\mathcal{M}}\right. \\
& +N_{f i}^{-}\left[-\frac{M_{f}+M_{i}}{2 \mathcal{M}}\left(W_{f}+W_{i}+M_{i}+M_{f}\right)\right. \\
& \left.\left.-\frac{\left(\omega_{f}+\omega_{i}\right)\left(E_{f}+E_{i}\right)+p_{f}^{2}+p_{i}^{2}}{2 \mathcal{M}}\right]\right], \\
Z_{V}^{(C)}= & g_{P} P V f_{V} N_{f i}^{-} \frac{p_{f} p_{i}}{\mathcal{M}}, \\
X_{V}^{(\mathrm{SO})}= & g_{P} P V g_{V} N_{f i}^{-}\left[-\frac{\left(m_{f}^{2}-m_{i}^{2}\right)\left(M_{i}-M_{f}\right)}{m_{V}^{2}}\right. \\
& \left.-\left(W_{f}+W_{i}+M_{f}+M_{i}\right)\right]+g_{P P V} f_{V} N_{f i}^{-} \\
& \times\left[-\frac{M_{f}+M_{i}}{2 \mathcal{M}}\left(W_{f}+W_{i}+M_{f}+M_{i}\right)\right. \\
& \left.-\frac{\left(\omega_{f}+\omega_{i}\right)\left(E_{f}+E_{i}\right)+p_{f}^{2}+p_{i}^{2}}{2 \mathcal{M}}\right], \\
& {\left[\begin{array}{l}
g_{f} \\
p_{i}
\end{array}\right.} \\
& \\
&
\end{aligned}
$$$$
Y_{V}^{(\mathrm{SO})}=-g_{P P V} f_{V} N_{f i}^{-} \frac{p_{f} p_{i}}{\mathcal{M}} .
$$

(c). $J^{P}=2^{++}$tensor-meson exchange.

$$
\begin{aligned}
X_{T}^{(C)}= & -\frac{g_{P P T} F_{1}}{m_{\pi^{+}}} N_{f i}^{+}\left[A_{0}+\frac{B_{0}}{2}\left(W_{f}+W_{i}-M_{i}-M_{f}\right)\right], \\
Y_{T}^{(C)}= & -\frac{g_{P P T} F_{1}}{m_{\pi^{+}}}\left[N_{f i}^{+}\left[A_{1}+\frac{B_{1}}{2}\left(W_{f}+W_{i}-M_{f}-M_{i}\right)\right]\right. \\
& \left.+N_{f i}^{-}\left[-A_{0}+\frac{B_{0}}{2}\left(W_{f}+W_{i}+M_{f}+M_{i}\right)\right]\right], \\
Z_{T}^{(C)}= & -\frac{g_{P P T} F_{1}}{m_{\pi^{+}}}\left[N_{f i}^{+} A_{2}+N_{f i}^{-}\left[-A_{1}+\frac{B_{1}}{2}\right.\right. \\
& \left.\left.\times\left(W_{f}+W_{i}+M_{f}+M_{i}\right)\right]\right], \\
U_{T}^{(C)}= & \frac{g_{P P T} F_{1}}{m_{\pi^{+}}} N_{f i}^{-} A_{2},
\end{aligned}
$$

$X_{T}^{(\mathrm{SO})}=\frac{g_{P P T} F_{1}}{m_{\pi^{+}}} N_{f i}^{-}\left[-A_{0}+\frac{B_{0}}{2}\left(W_{f}+W_{i}+M_{i}+M_{f}\right)\right]$,

$Y_{T}^{(\mathrm{SO})}=\frac{g_{P P T} F_{1}}{m_{\pi^{+}}} N_{f i}^{-}\left[-A_{1}+\frac{B_{1}}{2}\left(W_{f}+W_{i}+M_{i}+M_{f}\right)\right]$,

$Z_{T}^{(\mathrm{SO})}=-\frac{g_{P P T} F_{1}}{m_{\pi^{+}}} N_{f i}^{-} A_{2}$. 
where $A_{0}, A_{1}, A_{2}, B_{0}$, and $B_{1}$ depend on the mass and momentum of the particles as follows:

$$
\begin{aligned}
A_{0}= & F_{2}\left[\left(\frac{\left(\omega_{i}+\omega_{f}\right)\left(E_{f}+E_{i}\right)+p_{f}^{2}+p_{i}^{2}}{2}\right)^{2}\right. \\
& +\frac{1}{4 m_{T}^{4}}\left(m_{f}^{2}-m_{i}^{2}\right)^{2}\left(M_{i}^{2}-M_{f}^{2}\right)^{2} \\
& -\frac{\left(\omega_{i}+\omega_{f}\right)\left(E_{f}+E_{i}\right)+p_{f}^{2}+p_{i}^{2}}{2 m_{T}^{2}}\left(m_{f}^{2}-m_{i}^{2}\right) \\
& \times\left(M_{i}^{2}-M_{f}^{2}\right)-\frac{1}{3}\left(-2\left(m_{f}^{2}+m_{i}^{2}\right)+\frac{\left(m_{f}^{2}-m_{i}^{2}\right)^{2}}{m_{T}^{2}}\right. \\
& \left.+\frac{\left(E_{f}-E_{i}\right)^{2}+\left(\omega_{f}-\omega_{i}\right)^{2}}{2}-p_{f}^{2}-p_{i}^{2}\right) \\
& \times\left(\frac{\left(M_{i}^{2}-M_{f}^{2}\right)^{2}}{4 m_{T}^{2}}-\frac{M_{f}^{2}+M_{i}^{2}}{2}+\frac{1}{4}\left(-p_{f}^{2}-p_{i}^{2}\right.\right. \\
& \left.\left.\left.+\frac{\left(E_{f}-E_{i}\right)^{2}+\left(\omega_{f}-\omega_{i}\right)^{2}}{2}\right)\right)\right] \\
& +\left[-\frac{\left(\omega_{i}+\omega_{f}\right)\left(E_{f}+E_{i}\right)+p_{f}^{2}+p_{i}^{2}}{2 m_{T}^{2}}\left(m_{f}^{2}-m_{i}^{2}\right)\right. \\
& \left.\times\left(\frac{\left(M_{i}^{2}-M_{f}^{2}\right)}{2 m_{T}^{2}}\left(M_{i}-M_{f}\right)-\frac{1}{2}\left(M_{f}+M_{i}\right)\right)\right] \\
& \times\left(M_{i}-M_{f}\right)+\frac{1}{2 m_{T}^{4}}\left(m_{f}^{2}-m_{i}^{2}\right)^{2}\left(M_{i}^{2}-M_{f}^{2}\right) \\
& +\frac{1}{3}\left(-2\left(m_{f}^{2}+m_{i}^{2}\right)+\frac{\left(m_{f}^{2}-m_{i}^{2}\right)^{2}}{m_{T}^{2}}\right.
\end{aligned}
$$

$$
\begin{aligned}
A_{1}= & \frac{F_{2}}{F_{1}}\left[\left(\omega_{i}+\omega_{f}\right)\left(E_{f}+E_{i}\right)+p_{f}^{2}+p_{i}^{2}\right. \\
& -\frac{\left(m_{f}^{2}-m_{i}^{2}\right)\left(M_{i}^{2}-M_{f}^{2}\right)}{m_{T}^{2}}-\frac{1}{6}\left(-2\left(m_{f}^{2}+m_{i}^{2}\right)\right. \\
& +\frac{\left(m_{f}^{2}-m_{i}^{2}\right)^{2}}{m_{T}^{2}}+\frac{\left(E_{f}-E_{i}\right)^{2}+\left(\omega_{f}-\omega_{i}\right)^{2}}{2} \\
& \left.-p_{f}^{2}-p_{i}^{2}\right)-\frac{\left(M_{i}^{2}-M_{f}^{2}\right)^{2}}{6 m_{T}^{2}}+\frac{M_{f}^{2}+M_{i}^{2}}{3} \\
& \left.-\frac{1}{6}\left(\frac{\left(E_{f}-E_{i}\right)^{2}+\left(\omega_{f}-\omega_{i}\right)^{2}}{2}-p_{f}^{2}-p_{i}^{2}\right)\right] p_{f} p_{i} \\
& +\left[-\frac{\left(m_{f}^{2}-m_{i}^{2}\right)\left(M_{i}-M_{f}\right)}{m_{T}^{2}}\right. \\
& \left.-\frac{\left(M_{i}^{2}-M_{f}^{2}\right)\left(M_{i}-M_{f}\right)}{3 m_{T}^{2}}+\frac{M_{f}+M_{i}}{3}\right] p_{f} p_{i},
\end{aligned}
$$$$
A_{2}=-\frac{1}{3} p_{f}^{2} p_{i}^{2} \frac{F_{2}}{F_{1}},
$$$$
B_{0}=\left(\omega_{i}+\omega_{f}\right)\left(E_{f}+E_{i}\right)+p_{f}^{2}+p_{i}^{2}
$$$$
-\frac{\left(m_{f}^{2}-m_{i}^{2}\right)\left(M_{i}^{2}-M_{f}^{2}\right)}{m_{T}^{2}},
$$$$
B_{1}=2 p_{f} p_{i} .
$$

[1] R. A. Arndt, I. I. Strakovsky, R. L. Workman, and M. M. Pavan, Phys. Rev. C 52, 2120 (1995).

[2] R. A. Arndt, W. J. Briscoe, I. I. Strakovsky, R. L. Workman, and M. M. Pavan, Phys. Rev. C 69, 035213 (2004).

[3] R. Koch and E. Pietarinen, Nucl. Phys. A336, 331 (1980); J. R. Carter, D. V. Bugg, and A. A. Carter, ibid. B58, 378 (1973).

[4] CNS DAC (SAID), http://gwdac.phys.gwu.edu/

[5] J. S. Hyslop, R. A. Arndt, L. D. Roper, and R. L. Workman, Phys. Rev. D 46, 961 (1992).

[6] T. Nakano et al., Phys. Rev. Lett. 91, 012002 (2003).

[7] L. Maiani, G. Pancheri, and N. Paver, The Second DAФNE Physics Handbook (INFN, Frascati, 1995).

[8] Y. Akaishi and T. Yamazaki, Phys. Rev. C 65, 044005 (2002).

[9] M. Iwasaki, T. Suzuki, H. Bhang, G. Franklin, K. Gomikawa, R. Hayano, T. Hayashi, K. Ishikawa, S. Ishimoto, K. Itahashi,
T. Katayama, Y. Kondo, Y. Matsuda, T. Nakamura, S. Okada, H. Outa, B. Quinn, M. Sato, M. Shindo, H. So, T. Sugimoto, P. Strasser, K. Suzuki, S. Suzuki, D. Tomono, A. M. Vinodkumar, E. Widmann, T. Yamazaki, and T. Yoneyama arXiv:nuclex/0310018.

[10] M. M. Nagels, T. A. Rijken, and J. J. de Swart, Phys. Rev. D 17, 768 (1978); P. M. M. Maessen, T. A. Rijken, and J. J. de Swart, Phys. Rev. C 40, 2226 (1989).

[11] S. Weinberg, Physica A 96, 327 (1979); A. Manohar and H. Georgi, Nucl. Phys. B234, 189 (1984); H. Georgi, Annu. Rev. Nucl. Part. Sci. 43, 209 (1993).

[12] D. A. Liberman, Phys. Rev. D 16, 1542 (1977); J. E. F. T. Ribeiro, Z. Phys. C 5, 27 (1980); Y. Fujiwara, C. Nakamoto, and Y. Suzuki, Phys. Rev. C 54, 2180 (1996).

[13] J. Polchinski, Nucl. Phys. B231, 269 (1984).

[14] E. E. Salpeter and H. A. Bethe, Phys. Rev. 84, 1232 (1951). 
[15] V. G. Kadyshevsky, Sov. Phys. JETP 19, 443 and 597 (1964); Nucl. Phys. B6, 125 (1968); V. G. Kadyshevsky and M. D. Mateev, Nuovo Cimento A 55, 275 (1968); C. Itzykson, V. G. Kadyshevsky, and I. T. Todorov, Phys. Rev. D 1, 2823 (1970).

[16] H. Polinder and T. A. Rijken, paper II (in preparation).

[17] H. P. Stapp, T. J. Ypsilantis, and M. Metropolis, Phys. Rev. 105, 302 (1957).

[18] P. D. B. Collins and E. J. Squires, Regge poles in particle physics, Springer tracts in modern physics, Vol. 45 (SpringerVerlag, Berlin, 1968).

[19] H. Pilkuhn, The Interactions of Hadrons (North-Holland, Amsterdam, 1967).

[20] J. D. Bjorken and S. D. Drell, Relativistic Quantum Fields (McGraw-Hill, New York, 1965). We follow the conventions of this reference except for two things. First, we use Dirac spinors with the normalization $u^{\dagger}(\mathbf{p}) u(\mathbf{p})=2 E(\mathbf{p})$, in accordance with the normalization given by Eq. (2.13). Second, we have a (-) sign in the definition of the $M$ matrix [Eq. (2.2)].

[21] M. M. Nagels, T. A. Rijken, and J. J. de Swart, Phys. Rev. D 15, 2547 (1977).

[22] R. Blankenbecler and R. Sugar, Phys. Rev. 142, 1051 (1966); M. H. Partovi and E. L. Lomon, Phys. Rev. D 2, 1999 (1970); R. H. Thompson, ibid. 1, 110 (1970); A. A. Logunov and A. N. Tavkhelidze, Nuovo Cimento 29, 380 (1963); A. Gersten, P. A. Verhoeven, and J. J. de Swart, Nuovo Cimento A 26, 375 (1975).

[23] P. A. Verhoeven, Ph.D. thesis, University of Nijmegen, 1976.

[24] T. A. Rijken, Ann. Phys. (NY) 164, 1 (1985); 164, 23 (1985).
[25] F. E. Low, Phys. Rev. D 12, 163 (1975); S. Nussinov, Phys. Rev. Lett. 34, 1286 (1975).

[26] P. A. Carruthers, Spin and Isospin in Particle Physics (Gordon and Breach, New York, 1971). The isospin-1/2 isospin-3/2 transition operator we use is equal to $1 / \sqrt{2}$ times the operator defined in this reference.

[27] J. R. Taylor, Scattering Theory: The Quantum Theory on Nonrelativistic Collisions (Wiley, New York, 1972). In the SYM convention [17] the configuration space basic JLS states are $\mathcal{Y}_{J L S}^{M}(\hat{\mathbf{r}})=C_{m \mu M}^{L S J} Y_{m}^{L}(\hat{\mathbf{r}}) \chi_{\mu}^{(S)}$. Transformation to momentum space gives Eq. (A9).

[28] A. R. Edmonds, Angular Momentum in Quantum Mechanics (Princeton University Press, Princeton, 1957), The explicit relation between our $9 j$ symbols and those of this reference [Eq. (6.4.4)] is [31]

$$
\begin{aligned}
{\left[\begin{array}{lll}
j_{11} & j_{12} & j_{13} \\
j_{21} & j_{22} & j_{23} \\
j_{31} & j_{32} & j_{33}
\end{array}\right]=} & {\left[\left(2 j_{13}+1\right)\left(2 j_{31}+1\right)\left(2 j_{23}+1\right)\right.} \\
& \left.\times\left(2 j_{32}+1\right)\right]^{1 / 2}\left\{\begin{array}{lll}
j_{11} & j_{12} & j_{13} \\
j_{21} & j_{22} & j_{23} \\
j_{31} & j_{32} & j_{33}
\end{array}\right\} .
\end{aligned}
$$

[29] H. Polinder, Ph.D. thesis, University of Nijmegen, 2004, http:// nn-online.org/eprints/pdf/04.01.pdf or http://webdoc.ubn.kun. $\mathrm{nl} / \mathrm{mono} / \mathrm{p} /$ polinder_h/stromein.pdf.

[30] S. Gasiorowicz, Elementary Particle Physics (Wiley, New York, 1966).

[31] L. J. A. M. Somers, Ph.D. thesis, University of Nijmegen, 1984. 